\title{
Dual integral porosity shallow water model for urban flood modelling
}

\author{
Vincent Guinot (1,2), Brett F. Sanders (3), Jochen E. Schubert (3)
}

1: Université de Montpellier, UMR HSM, CC57, 163 rue Auguste Broussonnet, 34090 Montpellier, France

2: Inria Lemon, Bât 5 - CC05 017, 860 rue Saint-Priest, 34095 Montpellier Cedex 5, France

3: Department of Civil and Environmental Engineering, University of California, 4130 Engineering Gateway, Irvine, CA 92697-2175, USA

\begin{abstract}
With CPU times 2 to 3 orders of magnitude smaller than classical shallow water-based models, the shallow water equations with porosity are a promising tool for large-scale modelling of urban floods. In this paper, a new model formulation called the Dual Integral Porosity (DIP) model is presented and examined analytically and computationally with a series of benchmark tests. The DIP model is established from an integral mass and momentum balance whereby both porosity and flow variables are defined separately for control volumes and boundaries, and a closure scheme is introduced to link control volume- and boundary-based flow variables. Previously developed Integral Porosity (IP) models were limited to a single set of flow variables. A new transient momentum dissipation model is also introduced to account for the effects of sub-grid scale wave action on porosity model solutions, effects which are validated by fine-grid solutions of the classical shallow-water equations and shown to be important for achieving similarity in dam-break solutions. One-dimensional numerical test cases show that the proposed DIP model outperforms the IP model, with significantly improved wave propagation speeds, water depths and discharge calculations. A two-dimensional field scale test case shows that the DIP model performs better than the IP model in mapping the floods extent and is slightly better in reproducing the anisotropy of the flow field when momentum dissipation parameters are calibrated.
\end{abstract}

\section{Introduction}

Since their inception in the nineteen-nineties [10], shallow water models with porosity have arisen as a promising formalism for the inclusion of subgrid-scale topographical and geometric features [9, $4]$ in two-dimensional flow simulations. Their usefulness to tackle urban flood modelling has been illustrated by a number of applications, with reported CPU acceleration factors of two to three orders of magnitude compared to usual two-dimensional models solving the shallow water equations [20, $15,22,24,30,33,27]$. The shallow water equations with porosity have also motivated a number of numerical developments $[7,8,11,20,24]$.

The first versions of shallow water models with porosity for simulating urban floods incorporated a single porosity [20, 21]. Although the discretization of geometry-induced source terms in the Single Porosity (SP) model is more complex than for the usual shallow water equations $[8,11,20,24]$, the wave propagation speeds are identical. At the time the first finite volume discretization of the SP model was published [20], it did not fully account for the effect of flow path obstruction by the buildings in

(C) 2017. This manuscript version is made available under the Elsevier user license

http://www.elsevier.com/open-access/userlicense/1.0/ 
addition to the storage effect. It was thus proposed that two different porosities should be used: a domain-based porosity to account for mass and momentum storage and a boundary-based, connectivity porosity to account for mass and momentum transport [24]. An eigenvalue analysis of the resulting system showed that wave propagation speeds [24] (and the behaviour of the flow solutions) should be significantly different from those of the SP model. However, no practical guidelines could be provided to infer the connectivity porosity from the urban geometry. Independently from this research, the Integral Porosity (IP) model was proposed almost at the same time and published shortly afterwards [30]. The two key issues brought to the fore by [30] are that (i) the SP model is based on the implicit assumption that the Reference Elementary Volume (REV) [6] exists in the urban environment, (ii) the SP formalism is essentially isotropic. The IP model allows these two drawbacks to be eliminated because the integral formulation makes the connectivity porosity a local, directional, and deterministic descriptor based on the intersection of the computational mesh with flow obstructions, and the storage porosity is also given deterministically. This limited the need for calibration to directional building drag parameters to achieve closure in field-scale applications, whereas SP modelling studies had also examined the calibration of porosity [33]. Recently, an IP model that accounts for depth-dependent porosity has been developed as a framework for both subgrid topographic variability and blockage features such as buildings [27].

A one-dimensional analysis of the wave propagation speeds of the differential form of the IP model equations [18] confirms that the IP model structure is similar to the differential formulation proposed in [24]. Nevertheless, numerical experiments reported in a recent publication [28] and in the present paper show that the wave propagation speeds and discharge predictions in the IP model are significantly different from those in refined wave propagation simulations. The wave propagation speeds are the eigenvalues of the Jacobian matrix of the flux vector with respect to the conserved variable vector. Consequently, erroneous wave speeds usually reflect erroneous mass and/or momentum flux models. In this paper, the introduction of a dual set of flow variables corresponding to integrals over cells and boundaries, and a closure scheme which links the two solutions through the dual porosity definition, is shown to resolve this problem. The resulting Dual Integral Porosity (DIP) model supports a significant improvement in accuracy in verification test cases, and shows advantages compared to the IP model in a field-scale test problem.

The remainder of the paper is organised as follows. Section 2 presents the DIP formalism along with the domain-boundary closure model and a modification of the momentum fluxes to account for transient momentum dissipation which cannot be modelled like friction with a sink term. Section 3 presents the verification of the DIP model in idealized configurations where analytical solutions are known, as well as comparisons to SP and IP models. Section 4 presents a two-dimensional field scale test where the practical utility and versatility of the model is validated, i.e., ability to control anisotropic solutions through calibrated parameters. Sections 5 and 6 are devoted to a discussion and conclusions, respectively.

\section{$2 \quad$ Integral porosity shallow water models}

\subsection{Original formulation}

The conservation form of the model is obtained from a mass and momentum balance applied to a shallow water domain $\Omega$ with boundary $\Gamma$. The domain is partitioned into two regions: a solid region occupied by buildings, and a water region. Mass and momentum conservation leads to [30]:

$$
\begin{gathered}
\partial_{t} \int_{\Omega} \varepsilon h \mathrm{~d} \Omega+\int_{\Gamma} \varepsilon \mathbf{q} \cdot \mathbf{n} \mathrm{d} \Gamma=0 \\
\partial_{t} \int_{\Omega} \varepsilon \mathbf{q} \mathrm{d} \Omega+\int_{\Gamma} \varepsilon\left[(\mathbf{u . n}) \mathbf{q}+\frac{g}{2} h^{2} \mathbf{n}\right] \mathrm{d} \Gamma=\int_{\Omega} \varepsilon \mathbf{s}_{\Omega} \mathrm{d} \Omega+\int_{\Gamma} \varepsilon \mathbf{s}_{\Gamma} \mathrm{d} \Gamma \\
\mathbf{q}=h \mathbf{u}=\left[\begin{array}{c}
h u \\
h v
\end{array}\right], \mathbf{s}_{\Gamma}=\frac{g}{2} h_{\eta_{0}}^{2} \mathbf{n}
\end{gathered}
$$




$$
\mathbf{s}_{\Omega}=-g\left(h-h_{\eta_{0}}\right) \nabla z_{b}-\left(c_{D}^{b}+c_{D}^{f}\right)|\mathbf{u}| \mathbf{u}
$$

where $\mathbf{n}$ is the normal unit vector, $\mathbf{q}$ and $\mathbf{u}$ are respectively the unit discharge and flow velocity vectors, $\mathbf{s}_{\Omega}$ and $\mathbf{s}_{\Gamma}$ are respectively the domain and boundary source terms, $h$ is the water depth, $u$ and $v$ are respectively the $x$ - and $y$-velocities, $z_{b}$ is the bottom level, $\varepsilon$ is the porosity indicator (equal to 0 in the solid areas and 1 in the water region), $\eta_{0}$ is the average free surface elevation over the domain $\Omega, h_{\eta_{0}}=\eta_{0}-z_{b}, c_{D}^{b}$ and $c_{D}^{f}$ are respectively the drag coefficients for the building and bottom friction-induced stresses. The porosity over the sets $\Omega, \Gamma$ is defined as

$$
\phi_{S} \equiv \frac{\int_{S_{w}} \mathrm{~d} S}{\int_{S} \mathrm{~d} S}=\int_{S} \varepsilon(x, y) \mathrm{d} S, \quad S=\Omega, \Gamma
$$

As stressed in [30], the water storage porosity $\phi_{\Omega}$ and the water conveyance porosity $\phi_{\Gamma}$ are different in the general case. By definition, $\phi_{\Gamma}$ scales transport capacity and should be taken smaller than $\phi_{\Omega}$. The difference $\phi_{\Omega}-\phi_{\Gamma}$ thus represents an estimate for the fraction of the domain that does not contribute to transport. The constraint $\phi_{\Gamma} \leq \phi_{\Omega}$ is further substantiated by a wave propagation speed analysis, see Section 2.5.

Although questionable when a Reference Elementary Volume does not exist [30], the differential form of the equations for this model allows the behaviour of the solutions to be analysed. In the limit of an infinitesimal control volume $\Omega$, using the divergence theorem leads to a system in the form [24]

$$
\begin{gathered}
\partial_{t}\left(\phi_{\Omega} h\right)+\nabla \cdot\left(\phi_{\Gamma} \mathbf{q}\right)=0 \\
\partial_{t}\left(\phi_{\Omega} \mathbf{q}\right)+\nabla \cdot\left(\phi_{\Gamma} \mathbf{F}\right)=\phi_{\Omega} \mathbf{s}_{\Omega}+\nabla \cdot\left(\phi_{\Gamma} \mathbf{s}_{\Gamma}\right) \\
\mathbf{F}=\left[\begin{array}{cc}
h u^{2}+\frac{g}{2} h^{2} & h u v \\
h u v & h v^{2}+\frac{g}{2} h^{2}
\end{array}\right]=\mathbf{u} \otimes \mathbf{u}+\frac{g}{2} h^{2} \mathbf{I}
\end{gathered}
$$

where $\mathbf{I}$ is the identity matrix. The wave propagation speeds in the one-dimensional configuration are the eigenvalues of the system $[18,24]$ :

$$
\lambda_{1}=\frac{\phi_{\Gamma}}{\phi_{\Omega}}(u-c), \lambda_{2}=\frac{\phi_{\Gamma}}{\phi_{\Omega}} u, \lambda_{3}=\frac{\phi_{\Gamma}}{\phi_{\Omega}}(u+c), c=(g h)^{\frac{1}{2}}
$$

This system is hyperbolic. Its eigenvalues are those of the classical shallow water equations, multiplied by the ratio $\frac{\phi_{\Gamma}}{\phi_{\Omega}}$. The particular case $\phi_{\Gamma}=\phi_{\Omega}$ yields the wave propagation speeds of the classical Saint Venant equations. This property is used in finite volume discretizations of the single porosity equations to derive approximate Riemann solvers $[8,11,20]$. If the mesh is designed such that $\phi_{\Gamma}>\phi_{\Omega}$, the wave propagation speeds are larger than those of the classical shallow water equations. Such a behaviour is non-physical.

\subsection{Dual integral porosity model formulation}

The DIP model overcomes ambiguity in the definition of the fluid velocity, $\mathbf{u}$, at domain boundaries (versus within domain boundaries) with the introduction of dual flow variables $\left(\mathbf{u}_{\Omega}, \mathbf{u}_{\Gamma}\right)$ and substitution into equations (1a-b) as follows,

$$
\begin{gathered}
\partial_{t} \int_{\Omega} \varepsilon h_{\Omega} \mathrm{d} \Omega+\int_{\Gamma} \varepsilon \mathbf{q}_{\Gamma} \cdot \mathbf{n} \mathrm{d} \Gamma=0 \\
\partial_{t} \int_{\Omega} \varepsilon \mathbf{q}_{\Omega} \mathrm{d} \Omega+\int_{\Gamma} \varepsilon\left[\left(\mathbf{u}_{\Gamma} \cdot \mathbf{n}\right) \mathbf{q}_{\Gamma}+\frac{g}{2} h_{\Gamma}^{2} \mathbf{n}\right] \mathrm{d} \Gamma=\int_{\Omega} \varepsilon \mathbf{s}_{\Omega} \mathrm{d} \Omega+\int_{\Gamma} \varepsilon \mathbf{s}_{\Gamma} \mathrm{d} \Gamma
\end{gathered}
$$

For the number of equations to match the number of unknowns, a closure model is needed between the domain-averaged variable vector $\mathbf{u}_{\Omega}$ and the boundary-averaged variable vector $\mathbf{u}_{\Gamma}$. 
Closure model for the unit discharge. Consider an elemental boundary segment $\mathrm{d} \Gamma=[\mathrm{AB}]$ (Figure 1). Typically, $[\mathrm{AB}]$ is the edge of a computational cell in a finite volume or finite element discretization. The total discharge across $[\mathrm{AB}]$ is

$$
Q_{\mathrm{AB}}=\phi_{\Gamma} \mathbf{q}_{\Gamma} \cdot \mathbf{n} \mathrm{d} \Gamma
$$

Consider now the segment $\left[\mathrm{A}^{\prime} \mathrm{B}^{\prime}\right]$, obtained by shifting $[\mathrm{AB}]$ into the domain $\Omega$. For an infinitesimal shift, the discharge across $\left[\mathrm{A}^{\prime} \mathrm{B}^{\prime}\right]$ is equal to the discharge across $[\mathrm{AB}]$. It must be computed using the areal porosity $\phi_{\Omega}$ because the segment is located within the domain

$$
Q_{\mathrm{A}^{\prime} \mathrm{B}^{\prime}}=\phi_{\Omega} \mathbf{q}_{\Omega} \cdot \mathbf{n} \mathrm{d} \Gamma
$$

\section{Figure 1 about here}

Figure 1: Closure model for the unit discharge. Definition sketch.

Continuity requires that $Q_{\mathrm{AB}}=Q_{\mathrm{A}^{\prime} \mathrm{B}^{\prime}}$. This leads to the sufficient condition

$$
\mathbf{q}_{\Gamma}=\frac{\phi_{\Omega}}{\phi_{\Gamma}} \mathbf{q}_{\Omega} \Leftrightarrow \mathbf{u}_{\Gamma}=\frac{\phi_{\Omega}}{\phi_{\Gamma}} \frac{h}{h_{\Gamma}} \mathbf{u}_{\Omega}
$$

Note that modifying only the normal component of the flow velocity would suffice to satisfy continuity. However, doing so would make the direction of $\mathbf{q}_{\Gamma}$ artificially dependent on the direction of the interface. Therefore, both the normal and tangent unit discharges are modified by the same factor. With this correction, the mass flux across the boundary verifies

$$
\int_{\Gamma} \phi_{\Gamma} \mathbf{q}_{\Gamma} \cdot \mathbf{n} \mathrm{d} \Gamma=\int_{\Gamma} \phi_{\Omega} \mathbf{q}_{\Omega} \cdot \mathbf{n} \mathrm{d} \Gamma
$$

Closure model for the water depth. Substituting the closure model (14) into the expression for the momentum flux leads to

$$
\int_{\Gamma} \phi_{\Gamma}\left(\mathbf{u}_{\Gamma} \cdot \mathbf{n}\right) \mathbf{q}_{\Gamma} \mathrm{d} \Gamma=\frac{\phi_{\Omega}^{2}}{\phi_{\Gamma}} \int_{\Gamma} \frac{h_{\Omega}}{h_{\Gamma}}\left(\mathbf{u}_{\Omega} \cdot \mathbf{n}\right) \mathbf{q}_{\Omega} \mathrm{d} \Gamma
$$

A model must be proposed for the ratio $\frac{h_{\Omega}}{h_{\Gamma}}$ in the momentum equation. The simplest possible model, $h_{\Gamma}=h_{\Omega}$, is retained. It is based on the assumption that building-induced flow obstruction has negligible effects on the free surface elevation and that the reduction in the flow cross-sectional area is fully balanced with an increase in the flow velocity. This is a reasonable approximation for small values of the Froude number, based on gradually varied flow theory, and experiments have shown that supercritical (high Froude) flow impinging on clusters of buildings causes upstream shock waves that surround buildings by locally subcritical (low Froude) flow [35]. The expression for the momentum flux becomes

$$
\int_{\Gamma} \phi_{\Gamma}\left(\mathbf{u}_{\Gamma} \cdot \mathbf{n}\right) \mathbf{q}_{\Gamma} \mathrm{d} \Gamma=\frac{\phi_{\Omega}^{2}}{\phi_{\Gamma}} \int_{\Gamma}\left(\mathbf{u}_{\Omega} \cdot \mathbf{n}\right) \mathbf{q}_{\Omega} \mathrm{d} \Gamma
$$

\subsection{Accounting for transient momentum dissipation}

Solving the frictionless shallow water equations over street networks with a constant street width in the presence of piecewise constant initial and/or boundary conditions is shown in [15] to yield solutions with an almost perfectly self-similar $(x, t)$ behaviour. The waves in the solution are seen to propagate at a constant speed, making the solution at the scale of the street spacing dependent on the ratio $x / t$. The self-similarity of the solutions is not exact in that a street network with fixed width and spacing is not invariant when the $x$-coordinate is transformed, but self-similarity is observed for the 
solutions averaged on the scale of a building block. Two such examples are presented in [15]. The first deals with the propagation of a flood bore into an idealized urban network. The second consists of an urban dambreak problem. As illustrated by Section 3, the numerical solutions obtained by solving the frictionless shallow water equations on a fine grid cannot be reproduced by a porosity model that does not incorporate a momentum dissipation mechanism of some kind. Using the proposed DIP model without friction will lead to wrongly estimated wave propagation speeds, water depths and flow velocities, especially in the case of positive waves (rising water levels).

An explanation for this behaviour was introduced in [15]: when a positive wave propagates into a built area (Figure 2), multiple wave reflections occur against the building walls and generate moving bores (Figure 2, top). The wave reflections generate reactions to the hydrostatic pressure forces from the building walls. In Figure 2, the reaction from wall $\mathrm{W}_{2}$ is larger than that from wall $\mathrm{W}_{1}$ because the reflected bore is deeper (Figure 2, bottom). In the same way, the reaction from wall $\mathrm{W}_{4}$ is larger than that from wall $\mathrm{W}_{3}$. The resulting force is opposed to the average flow velocity and thus contributes to dissipate momentum. Steady state flow and decreasing water levels do not generate bores and no significant dissipation occurs due to wave reflection. This mechanism was confirmed in [15] by a detailed inspection of the water depth, velocity and hydraulic head fields in the refined shallow water simulations. Although the dissipation is not instantaneous in reality, it is assumed to occur on a time scale smaller than or of the same order of magnitude as the time scale needed by the wave to travel between two successive building rows. The assumption of instantaneous dissipation is thus a convenient approximation. The tests presented in Section 3 indicate that the proposed model is reasonably accurate even when the flow is supercritical.

\section{Figure 2 about here}

Figure 2: Propagation of a positive wave into a built area. Top: plan view of wave propagation and bore reflection. Bottom: side view along transect $[\mathrm{AB}]$. The dashed line indicates the initial water level.

Classical head loss models that are direct functions of the flow velocity are well-known to not allow for self-similar solutions in the case of piecewise constant initial/boundary value problems, and thus cannot reproduce the losses from subgrid-scale bores described above. The only possibility to retain the self-similar character of the solutions is for the momentum dissipation model to be divergencefree. In other words, the governing equations must consist only in a modification of the momentum flux and/or the conserved variable (e.g. by introducing a so-called rigidity, or inertia matrix). The proposed momentum dissipation model satisfies this condition. It is derived from that proposed in the multiple porosity model [15] under the so-called Local Equilibrium Assumption (LEA). Consider again a positive wave entering the averaging volume shown in Figure 2. Within an infinitesimal time interval $\mathrm{d} t$, the total momentum in the averaging volume is subjected to a positive variation $\phi_{\Omega} \mathrm{d} \mathbf{q}_{\Omega}$, and if the fraction of transported momentum that is dissipated in dead zones is defined by $\mu \in[0,1]$, the net variation in the average momentum over the averaging volume is thus $(1-\mu) \phi_{\Omega} \mathrm{d} \mathbf{q}_{\Omega}$. This is equivalent to multiplying the divergence of the momentum fluxes by a factor $(1-\mu) \leq 1$. When the averaging volume is subjected to a zero or negative variation (momentum and volume are withdrawn), no dissipation occurs. Consequently, the above correction is applied only for a rising water depth, that is, when $\partial_{t} h>0$, or equivalently when the divergence of the flow field is negative $\nabla \cdot \mathbf{q}<0$.

The anisotropic character of most urban layouts calls for generalizing the proposed momentum dissipation term in the form of a second-order tensor:

$$
\mathbf{D}=\left[\begin{array}{ll}
\mu_{x x} & \mu_{x y} \\
\mu_{y x} & \mu_{y y}
\end{array}\right]=\mathbf{B}\left[\begin{array}{cc}
\mu_{1} & 0 \\
0 & \mu_{2}
\end{array}\right] \mathbf{B}^{-1} \mathrm{~d} \mathbf{q}_{\boldsymbol{\Omega}}, \quad \mathbf{B}=\left[\begin{array}{cc}
\cos \theta_{1} & \cos \theta_{2} \\
\sin \theta_{1} & \sin \theta_{2}
\end{array}\right]
$$

where $\theta_{1}$ and $\theta_{2}$ are the angles of the principal directions of the urban layout with the $x$-axis. Note that this does not necessarily result in a symmetric tensor. The infinitesimal momentum variation becomes

$$
(\mathbf{I}-\mathbf{D}) \phi_{\Omega} \mathrm{d} \mathbf{q}_{\Omega}
$$




\subsection{Anisotropic building drag model}

The proposed model also incorporates an improvement to the building drag force model. Although deemed of secondary importance compared to the closure and the momentum dissipation models presented in Subsections 2.2-3, the revised building drag model allows the accuracy of integral porosity models to be increased further. This is illustrated by the computational examples in Section 4 . The building drag model originally implemented in the IP formulation [30] uses the following momentum source term (equation (4)

$$
\mathbf{s}_{D}^{b}=-C_{D}^{b}|\mathbf{u}| \mathbf{u}
$$

Firstly, the source term be made depth-dependent. For a given flow velocity, building drag force can be expected to be proportional to the contact surface between the water and the buildings. The contact surface being proportional to the water depth, it is proposed that the source term be scaled by the water depth. Secondly, many urban street networks are observed to exhibit anisotropy, thus motivating a tensor-based description of the building drag terms. The following formula is thus proposed

$$
\mathbf{s}_{D}^{b}=-\mathbf{C}_{D}^{b} a h|\mathbf{u}| \mathbf{u}, \mathbf{C}_{D}^{b}=\left[\begin{array}{ll}
C_{D, x x}^{b} & C_{D, x y}^{b} \\
C_{D, y x}^{b} & C_{D, y y}^{b}
\end{array}\right]
$$

where $\mathbf{C}_{D}^{b}$ is a drag coefficient tensor and $a$ is a the frontal area parameter introduced in vegetationinduced drag models [26, 34]. The spatial distribution of frontal area has previously been estimated deterministically for the IP formulation, in conjunction with a scalar drag coefficient, using building footprint data characterizing the shape of flow obstructions, leaving the scalar drag coefficient as a calibration parameter [30,31]. However, with this approach, $a$ is directionally dependent based on the shape and arrangement of obstructions relative to the local flow direction, which is typically not known a priori. This mandates a slow and iterative process to first estimate the flow to identify the flow direction, second to estimate frontal area based on flow direction, and third to estimate the drag coefficient. Eq. (21) overcomes this problem by: (i) assuming that $a$ is a scalar representative of the directionally-averaged frontal area, and (ii) using a drag coefficient tensor, which systematically accounts for direction through calibration. Moreover, if there is no prior knowledge about the drag coefficient tensor values and no significant spatial trends in $a$, then $a$ can be set to unity and the lumped effects of frontal area and the drag coefficient can be absorbed by the drag coefficient tensor. This approach is adopted here because all test cases involve arrays of buildings that are effectively homogeneous at the scale of the porosity model solution.

\subsection{Final formulation and numerical procedure}

Incorporating the new closure model, the momentum dissipation terms and the revised building drag model into the mass and momentum balances lead to the following set of governing equations

$$
\begin{gathered}
\partial_{t} \mathbf{v}+\int_{\Gamma} \mathbf{M F n} \mathrm{d} \Gamma=\int_{\Omega} \varepsilon \mathbf{s}^{\prime}{ }_{\Omega} \mathrm{d} \Omega+\int_{\Gamma} \varepsilon \mathbf{s}_{\Gamma}^{\prime} \mathrm{d} \Gamma \\
\mathbf{v} \equiv \phi_{\Omega}\left[\begin{array}{c}
h \\
h u \\
h v
\end{array}\right], \mathbf{F} \equiv \phi_{\Gamma}\left[\begin{array}{cc}
\beta h u & \beta h v \\
\beta^{2} h u^{2}+\frac{g}{2} h^{2} & \beta^{2} h u v \\
\beta^{2} h u v & \beta^{2} h v^{2}+\frac{g}{2} h^{2}
\end{array}\right], \mathbf{M} \equiv\left[\begin{array}{ccc}
1 & 0 & 0 \\
0 & 1-\mu_{x x} & -\mu_{x y} \\
0 & -\mu_{y x} & 1-\mu_{y y}
\end{array}\right] \\
\mathbf{s}_{\Omega}^{\prime}=\left[\begin{array}{c}
g\left(h-h_{\eta_{0}}\right) S_{0, x}-c_{D}^{f}|\mathbf{u}| u \\
g\left(h-h_{\eta_{0}}\right) S_{0, y}-c_{D}^{f}|\mathbf{u}| v
\end{array}\right]-\left[\begin{array}{cc}
0 & 0 \\
C_{D, x x}^{b} & C_{D, x y}^{b} \\
C_{D, y x}^{b} & C_{D, y y}^{b}
\end{array}\right] a h|\mathbf{u}| \mathbf{u} \\
\mathbf{s}_{\Gamma}^{\prime}=\frac{g}{2} h_{\eta_{0}}^{2}\left[\begin{array}{c}
0 \\
n_{x} \\
n_{y}
\end{array}\right]
\end{gathered}
$$


where $n_{x}$ and $n_{y}$ are respectively the $x$ - and $y$-components of the normal unit vector to the boundary, $S_{0, x}=-\partial_{x} z_{b}$ and $S_{0, y}=-\partial_{y} z_{b}$ are respectively the $x$ - and $y$-bottom slopes. Note that the bottom friction term originally proposed in [30] is used in the DIP model too for the sake of model comparison.

The equations are discretized using an explicit finite volume approach $[8,11,15,20,30]$. The frictionless part of the equations and the friction-induced source terms are solved sequentially using a time splitting procedure.

Frictionless terms. The frictionless part of the equations is first solved as

$$
\begin{gathered}
\mathbf{v}_{i}^{n+1 / 2}=\mathbf{v}_{i}^{n}+\frac{\Delta t}{A_{i}} \sum_{j \in N(i)} L_{i j} \mathbf{M}_{i} \mathbf{F}_{i j} \cdot \mathbf{n}_{i j}+\Delta t \sum_{j \in N(i)} \mathbf{S}_{\Gamma, i j} \mathbf{n}_{i j} \\
\mathbf{S}=L_{i j}\left[\begin{array}{cc}
0 & 0 \\
s_{\Gamma, i j} & 0 \\
0 & s_{\Gamma, i j}
\end{array}\right] \\
s_{\Gamma, i j} \equiv-g\left[\frac{\phi_{\Omega, i}-\phi_{\Gamma, i j}}{2} h_{i}^{2}+\phi_{\Gamma, i j} \frac{\max \left(0, \eta_{i}-z_{b, j}\right)+\eta_{i}-z_{b, i}}{2} \max \left(0, \min \left(\eta_{i}, z_{b, j}\right)-z_{b, i}\right)\right]
\end{gathered}
$$

where the subscript $i$ is the cell number, the superscript $n$ denotes the time level, $N(i)$ is the set of neighbouring cells for the cell $i, A_{i}$ is the plan view area of the cell $i, L_{i j}$ and $\mathbf{n}_{i j}$ are respectively the length and normal unit vector of the interface between the cells $i$ and $j, \Delta t$ is the computational time step. The flow variables in equations (27-28) are cell-averaged quantities and should bear the subscript $\Omega$. It is omitted in the notation for the sake of clarity. The discretized source term $\mathbf{S}_{\Gamma, i j}$ accounts for the variations in the porosity and bottom levels across the interface. It thus incorporates the discretization of the source term $\mathbf{s}_{\Gamma}$ and the contribution of the bottom slope in $\mathbf{s}_{\Omega}$, as detailed in [11].

Friction terms. The solution is updated to account for the friction term using time splitting with an exact solution [15]. The flow variables are first rotated into the coordinates formed by the principal directions of the building drag tensor. In this coordinate system, the equations become

$$
\partial_{t} \phi_{\Omega}\left[\begin{array}{c}
h \\
h u_{1} \\
h u_{2}
\end{array}\right]=-\phi_{\Omega}\left[\begin{array}{c}
0 \\
\left(c_{D}^{f}+C_{D, 1}^{b} a h\right)|\mathbf{u}| u_{1} \\
\left(c_{D}^{f}+C_{D, 2}^{b} a h\right)|\mathbf{u}| u_{2}
\end{array}\right]
$$

where the subscripts 1 and 2 denote the component of the velocity along the wo principal directions. Simplifying by $\phi_{\Omega}$ leads to

$$
\partial_{t} h u_{p}=-\left(c_{D}^{f}+C_{D, p}^{b} a h\right)|\mathbf{u}| u_{p}, \quad p=1,2
$$

Under the assumption of small time steps, the equation is linearised into

$$
\partial_{t}\left(h u_{p}\right)_{i}=-\left(c_{D}^{f}+C_{D, p}^{b} a h\right)\left|\mathbf{u}_{i}^{n}\right| u_{p}, \quad p=1,2
$$

with analytical solution

$$
\begin{gathered}
h_{i}^{n+1}=h_{i}^{n+1 / 2} \\
\left(h u_{p}\right)_{i}^{n+1}=\exp \left[-\left(c_{D}^{f}+C_{D, p}^{b} a h\right)\left|\mathbf{u}_{i}^{n}\right| \Delta t\right]\left(h u_{p}\right)_{i}^{n+1 / 2}
\end{gathered}
$$


Remark on flux computation. The reader's attention is drawn to the computation of the fluxes in (27). The finite volume implementations presented in the literature $[11,8,15,20,30]$ use Riemann solvers to compute the flux tensor F. Noticing that the flux tensor in (??) can be rewritten as

$$
\mathbf{F} \equiv \phi_{\Gamma}\left[\begin{array}{cc}
h U & h V \\
h U^{2}+\frac{g}{2} h^{2} & h U V \\
h U V & h V^{2}+\frac{g}{2} h^{2}
\end{array}\right],\left[\begin{array}{l}
U \\
V
\end{array}\right] \equiv \beta\left[\begin{array}{l}
u \\
v
\end{array}\right]
$$

the structure of the flux tensor is identical to that of the classical two-dimensional shallow water equations. It stems as an obvious consequence that the fluxes can be computed using any standard Riemann solver for the classical 2D shallow water equation, by defining the left and right states of the Riemann problem as

$$
\mathbf{v}_{L} \equiv \phi_{\Omega, L}\left[\begin{array}{c}
h_{L} \\
\beta_{L} h_{L} u_{L} \\
\beta_{L} h_{L} v_{L}
\end{array}\right], \mathbf{v}_{R} \equiv \phi_{\Omega, L}\left[\begin{array}{c}
h_{R} \\
\beta_{R} h_{R} u_{R} \\
\beta_{R} h_{R} v_{R}
\end{array}\right]
$$

where $\phi_{\Omega, S}(S=L, R)$ denotes $\phi_{\Omega}$ on the side $S$ of the interface.

The discretization (27) being explicit, it is subjected to a stability constraint based on the CFL of the fastest waves. The CFL condition for an unstructured grid is given in [32] and will not be recalled here. The formulas for the wave propagation speeds are derived in the next section.

\subsection{Eigenvalue analysis}

For the sake of clarity, the one-directional analysis of the equations is presented. The flux vector $\mathbf{f}$ is thus the first column of the flux tensor $\mathbf{F}$. Retaining only the conservation part of the equations, applying the integral form $(10,11,17)$ to an infinitesimal control volume, applying the divergence theorem under the assumption of continuous and differentiable solutions leads to a system in the form

$$
\begin{gathered}
\partial_{t} \mathbf{v}+\partial_{x}(\mathbf{M f})=0 \\
\mathbf{v} \equiv \phi_{\Omega}\left[\begin{array}{c}
h \\
h u \\
h v
\end{array}\right], \mathbf{f} \equiv \phi_{\Omega}\left[\begin{array}{c}
h u \\
(1-\mu) \beta h u^{2}+(1-\mu) \frac{g}{2 \beta} h^{2} \\
(1-\mu) \beta h u v
\end{array}\right]=\phi_{\Omega}\left[\begin{array}{c}
h u \\
\eta h u^{2}+\frac{\eta}{\beta^{2}} \frac{g}{2} h^{2} \\
\eta h u v
\end{array}\right]
\end{gathered}
$$

where $\eta \equiv(1-\mu) \beta$. It is interesting to notice that only the storage porosity $\phi_{\Omega}$ appears in the continuity equation (in contrast with the original IP model). This makes the new continuity equation consistent with that derived by Defina [9]. Table 1 summarizes the various available formulations for $\mathbf{u}$ and $\mathbf{f}$, the corresponding Jacobian matrices $\mathbf{A}$ and their eigenvalues $\lambda$ in the one-dimensional case.

\section{Table 1 about here}

Table 1: Summary of the various formulations. For the sake of conciseness, only the one-dimensional version is presented. Note: $c \equiv(g h)^{1 / 2}, c^{\prime} \equiv \eta^{\frac{1}{2}}\left[(\eta-1) u^{2}+\frac{c^{2}}{\beta^{2}}\right]^{\frac{1}{2}}$.

The present dual formulation implicitly rules out the configuration $\phi_{\Gamma}>\phi_{\Omega}$. Assuming $\phi_{\Gamma}>\phi_{\Omega}$ induces $\beta<1$, which leaves room for situations where $\eta<1$. Then combinations $(c, u)$ exist such that $(\eta-1) u^{2}+\frac{c^{2}}{\beta^{2}}<0$ and the $\lambda_{1}$ and $\lambda_{3}$ are complex, inducing a loss of hyperbolicity and making the initial value problem ill-posed. This is a strong point in favour of the modified model in that meaningless porosity configurations are ruled out by assumption. Note that this is not the case with the IP model, that allows for $\beta<1$, although such a situation is non-physical (see Section 2.1). 
Table 2 about here

Table 2: Propagation of a positive wave. Overview of simulations. (C): parameter is calibrated; (F): parameter is fixed; (NA): Not Applicable because the parameter does not exist in the model.

\section{Verification Test Cases}

\subsection{Positive Wave}

The purpose of this test case is to verify the DIP model formulation including the transient dissipation model proposed in the previous section. It in inspired from [15]. A periodic, orthogonal street network is generated (Figure 3 , left). The spatial period in the longitudinal and transverse direction are respectively $L$ and $2 L$, with $L=100 \mathrm{~m}$. The street width is $W=50 \mathrm{~m}$ in both directions. The bottom is horizontal, friction is assumed negligible. The initial and boundary conditions are

$$
\begin{gathered}
\mathbf{v}(x, 0)=\left[h_{0}, 0,0\right]^{T} \quad \forall x>0 \\
{[h u, h v]^{T}(0, t)=\left[q_{b}, 0\right] \quad \forall t>0}
\end{gathered}
$$

where $h_{0}$ and $q_{b}$ are respectively the initial water depth and boundary unit discharge. A positive wave is generated by setting $q_{b}=1 \mathrm{~m}^{2} \mathrm{~s}^{-1}$ with $h_{0}=1 \mathrm{~m}$. The two-dimensional frictionless shallow water equations are solved numerically over a $2.5 \mathrm{~m} \times 2.5 \mathrm{~m}$ computational grid (Figure 3 , right). Symmetry considerations allow a complete solution to be obtained by meshing only half a period in the transverse direction, $0 \leq y \leq L$. When solving the porosity-based shallow water equations, a much coarser computational grid is used, with cell widths $(\Delta x, \Delta y)=(20 \mathrm{~m}, 100 \mathrm{~m})$.

\section{Figure 3 about here}

Figure 3: Propagation of a positive wave into an idealized urban area. Left: definition sketch for the street network. Right: bird's eye view of the free surface elevation computed at $t=600$ s over a $2.5 \mathrm{~m} \times 2.5 \mathrm{~m}$ computational grid.

Figure 4 shows side views of the free surface elevation profiles computed at $t=300 \mathrm{~s}$ and $t=600 \mathrm{~s}$. Several simulations are carried out (Table 2): (i) the classical shallow water model on the $2.5 \mathrm{~m}$ square grid, (ii) the IP model [30] without friction $\left(C_{D}^{b}=0\right)$, (iii) the DIP model with $\left(\mu_{x x}, C_{D}^{b}\right)=(0,0)$, (iv) DIP with $\left(\mu_{x x}, C_{D}^{b}\right)=(0,0.01)$ and (v) DIP with $\left(\mu_{x x}, C_{D}^{b}\right)=(0.156,0)$. Simulation (i) is considered as the reference simulation. The value $C_{D}^{b}=0.01$ used in Simulation (iv) is the numerical value for which the propagation speed of the centre of the bore is approximated best. The value $\mu_{x x}=0.156$ in Simulation (v) was calibrated to best reproduce both the average bore wave propagation speed and the water level at the model boundary. Note that, in the reference Simulation (i), the water level oscillates about an average value that is almost constant with $x$. The midpoint of the bore is located at $x=710 \mathrm{~m}$ for $t=300 \mathrm{~s}$ and $x=1400 \mathrm{~m}$ for $t=600 \mathrm{~s}$. This confirms the self-similar character of the reference solution at the scale of the network period $(L=100 \mathrm{~m})$.

\section{Figure 4 about here}

Figure 4: Propagation of a positive wave into an idealized urban network. Side views of the free surface profiles computed at $t=300 \mathrm{~s}$ and $t=600 \mathrm{~s}$.

As expected from the wave speed analysis, the IP model yields a significantly underestimated wave propagation speed, even when the head loss is assumed zero $\left(C_{D}^{b}=0\right)$. As a result, the positive wave in Simulation (ii) is slower and the free surface elevation is higher than the reference case, Simulation (i). Increasing $C_{D}^{b}$ would only slow down the wave propagation speed even more and is therefore 
not an option. The DIP model without transient dissipation (Simulation (iii)), in contrast, yields a slightly overestimated wave speed and a subsequently slightly underestimated free surface elevation. In Simulation (iv), tuning the building drag coefficient $C_{D}^{b}$ improves prediction of the bore speed but destroys the self-similar character of the flow solution, with a sloping free surface and a time-dependent free surface elevation at the boundary. Only Simulation (v) predicts the bore speed and boundary water level accurately while preserving the self-similar character of the solution.

\subsection{Negative Wave}

The purpose of this test is to validate the absence of transient momentum dissipation when $\partial_{t} h<0$, as formulated in the DIP model. The physical layout and initial/boundary conditions are identical to those in Section 3.2, with the exception that $q_{b}=-0.5 \mathrm{~m}^{2} \mathrm{~s}^{-1}$ in order to create a negative wave. The following simulations are carried out (Table 3): (i) the reference simulation whereby the classical 2D shallow water equations are solved on the $2.5 \mathrm{~m} \times 2.5 \mathrm{~m}$ refined grid, (ii) IP simulation without building drag $\left(C_{D}^{b}=0\right)$, (iii) DIP with $\left(\mu_{x x}, C_{D}^{b}\right)=(0.156,0)$, where the momentum dissipation tensor $\mathbf{D}$ is activated regardless of the sign of $\partial_{t} h$, (iv) DIP with a drag parametrization for building resistance but without transient dissipation $\left(\mu_{x x}, C_{D}^{b}\right)=(0,0.01)$, and (v) DIP with the momentum dissipation tensor active for only $\partial_{t} h>0$ (i.e., proposed model formulation). Note that $\partial_{t} h \leq 0$ in this test case, so momentum dissipation has no effect on the fluxes. The values $\mu_{x x}=0.156$ and $C_{D}^{b}=0.01$ are used in this test case because they appeared as the best fitting values in the previous test. Note that in Simulation (i), the locally dropping water level next to the left boundary is due to boundary effects and should be disregarded.

\section{Table 3 about here}

Table 3: Propagation of a negative wave.Overview of simulations. (C): parameter is calibrated; (F): parameter is fixed; (NA): Not Applicable because the parameter does not exist in the model.

\section{Figure 5 about here}

Figure 5: Propagation of a negative wave into an idealized urban network. Side views of the free surface profiles computed at $t=300 \mathrm{~s}$ and $t=600 \mathrm{~s}$.

As in the positive wave test case, the IP model (ii) yields significantly underestimated wave propagation speeds. Consequently, the boundary value for the free surface elevation is underestimated. Simulation (iii) shows that when dissipation is added to the DIP model for $\partial_{t} h \leq 0$, wave speeds and water levels are also underestimated in the region of constant state behind the wave. In Simulation (iv), the presence of a non-zero source term on the right-hand side of the momentum equation destroys the self-similar character of the solution. The water depth at the boundary decreases with time as a result of the increasing length over which friction occurs. The proposed DIP model formulation (Simulation (v)) is the only one where the self-similar character of the solution is preserved and the water levels and average location of the negative wave are accurately predicted. Comparing Simulations (iii) and (v) confirms that the proposed momentum dissipation model should be triggered only when the water level decreases.

Although Simulation (v) allows the average propagation speed of the negative wave to be estimated accurately, it fails to represent correctly the spreading of the wave. This failure is explained by inspecting the velocity field in the reference simulation (i). Figure 6 shows the velocity distribution for $725 \mathrm{~m} \leq x \leq 925 \mathrm{~m}$ at $t=600 \mathrm{~s}$. The figure clearly indicates that the flow velocity is not uniform across the street. Along the building walls, a low velocity region can be observed, with a width approximately $20 \%$ of the width of the street. In this low velocity region, the velocity is approximately twice as small as the velocity along the centreline of the street. This low velocity region is due to the water being abstracted from the lateral streets into the main street. The nearly immobile water coming from the 


\section{Figure 6 about here}

Figure 6: Propagation of a negative wave into an idealized urban network. Reference simulation(i). Velocity at $t=600 \mathrm{~s}$ next to the left-hand boundary.

\section{Table 4 about here}

Table 4: Urban dam-break problem. Overview of simulations. C: parameter is calibrated; F: parameter is fixed; NA: Not Applicable because the parameter does not exist in the model.

lateral street mixes with the fast flowing water in the main street, causing a decrease in the flow speed near the interface between the two street networks. This non-uniform velocity distribution is not accounted for in the proposed closure model (14), that assumes a uniform flow velocity over the cross-sections between the buildings. Improving the closure model (14) would require that a correction coefficient be applied to decrease $\phi_{\Gamma}$, which would result in increasing $\left\|\mathbf{u}_{\Gamma}\right\|$. Increasing $\left\|\mathbf{u}_{\Gamma}\right\|$ would indeed lead to increase $c^{\prime}$ (see formula in Table 1) and a subsequently increased spreading of the wave.

\subsection{Dam-Break}

The urban dam break problem was introduced in [15]. It is an initial value problem with a piecewise constant initial state solved over the periodic street network presented in Sections 3.1 and 3.2. It is a Riemann problem for the porosity-based equations. The initial conditions are defined as

$$
\begin{aligned}
& h(x, 0)= \begin{cases}h_{L} & \text { if } x<0 \\
h_{R} & \text { if } x>0\end{cases} \\
& \mathbf{u}(x, 0)= \\
& \quad 0 \forall x
\end{aligned}
$$

In the present test the street network extends from $x=-2 \mathrm{~km}$ to $x=+2 \mathrm{~km}$. The following simulations are carried out (Table 4): (i) classical 2D shallow water equations solved using the same refined grid as in Sections 3.1-2, (ii) single porosity (SP) model [20], (iii) IP model, (iv) DIP model with $\mu_{x x}=0.48$, (v) DIP model with $\mu_{x x}=0.41$. For $\mu_{x x}=0.48$ the propagation speed of the shock wave in the DIP model is the propagation speed of the middle of the shock in Simulation(i). For $\mu_{x x}=0.41$ the propagation speed of the shock wave in the porosity model is that of the head of the shock in Simulation (i). Figure 7 shows the water depth and unit discharge profiles computed at $t=200 \mathrm{~s}$. In Figure 7a the computational results of Simulation(i) are plotted for all of the computational cells. In Figures 8b-d, the results of Simulation (i) are averaged over the $100 \mathrm{~m}$ spatial period of the street network to produce a solution at the resolution of the porosity model, which filters out high frequency fluctuations associated with wave reflections off building walls. Quite expectedly, the SP model yields strongly overestimated wave propagation speeds (Figure 8a) and unit discharges (Figure 7b). The IP model produces strongly underestimated wave propagation speeds (Figure 7a) and overestimated unit discharges (Figure 7b). The DIP model, in contrast, accurately computes all aspects of the water depth and unit discharge profiles except the steepness of the forward moving shock wave (Figures $7 \mathrm{~b}, \mathrm{~d}$ ). Modifying the momentum dissipation coefficient $\mu_{x x}$ leads to different water depths and unit discharges behind the shock wave and does not affect the upstream part of the rarefaction wave, where no momentum dissipation occurs.

\section{Figure 7 about here}

Figure 7: Urban dam break problem. Comparison between the refined 2D shallow water simulation and the various models. (a) Water depth profiles, Simulation (i) displayed for all computational points. (b) Water depth profiles, Simulation (i) results are averaged over each $100 \mathrm{~m} \times 100 \mathrm{~m}$ building block. (c), (d): unit discharge profiles, Simulation (i) results are averaged over each 100m-long building block. 


\section{Field-Scale Levee-Break Flood Modelling}

\subsection{Simulation objectives and setup}

The purpose of this test case is validate field-scale applicability of the DIP model and to analyse and discuss model performance. The test case chosen here involves a hypothetical scenario whereby an earthen levee protecting a $450 \mathrm{~m} \times 650 \mathrm{~m}$ neighbourhood of West Sacramento (CA) suddenly fails, as shown in Figure 8. The Sacramento conurbation is one of many metropolitan areas around the world where vast areas of development are below sea level, protected by levees, and at risk of major damage and casualties in the unfortunate event of a levee failure e.g. [25]. Consequently, models that efficiently and accurately model the hazard are of great interest for emergency preparedness and response. For this hypothetical scenario, it is assumed that water in the Sacramento River Deep Water Ship Channel is at a stage of $8 \mathrm{~m}$ compared to an average ground elevation of $4 \mathrm{~m}$ East of the levee, and for simplicity, that the levee breaches instantaneously with a width of $100 \mathrm{~m}$ and a bottom elevation of $5 \mathrm{~m}$. Flooding dynamics are established by solving the classical shallow-water equations on a refined grid, and the IP and DIP models are run on specially designed coarse grids and compared to the refined grid solution to characterize porosity model errors [22]. Since the purpose is to analyse porosity model errors, as opposed to structural model errors associated with shallow-water models [22], a zero bottom friction is assumed in all models. Previous work has shown that in densely developed areas, the blockage effects of buildings are far more important than bottom friction relative to flooding predictions [31, 22].

\section{Figure 8 about here}

Figure 8: Real-World test case. Aerial view of the West Sacramento neighbourhood. (c) Google Earth. Neighbourhood centre coordinates: lat. $38^{\circ} 37^{\prime} 15^{\prime \prime} \mathrm{N}$, long. $121^{\circ} 34^{\prime} 44^{\prime \prime} \mathrm{W}$.

Aerial laser scanning point cloud data (lidar) were provided by the City of West Sacramento and processed using a combination of automated and manual techniques in ArcGIS (ESRI, Redlands, California, USA) to create a $3 \mathrm{~m}$ resolution Digital Terrain Model (DTM) and a $3 \mathrm{~m}$ resolution building mask raster with values of 0 where buildings are present and values of 1 where there are no buildings. This corresponds to the finest possible resolution considering the spacing of point cloud data within the study area. Building walls are modelled with a precision of approximately $\pm 1.5 \mathrm{~m}$ in the $\mathrm{E}-\mathrm{W}$ and N-S directions, and as such, closely aligned building walls are not always distinguished from each other leading to the fusing of multiple buildings into a single structure. Small gaps in flow barriers are often important considerations in the development of urban flood inundation models, and require groundbased surveys to properly document [13]. However, for the present study, errors in the approximation of building shape (as well as storage and conveyance) do not undermine the investigation because the refined solution by the classical shallow-water equations and the coarse grid solution by the porosity models reference the same building data.

\subsection{Models}

Fine grid model. The classical shallow-water equations are solved on a refined grid consisting of an unstructured mesh of triangular and quadrilateral cells that is constrained by building walls. This represents the so-called building-hole method of representing buildings in urban flood models [31]. The mesh consists of 78840 cells with an average cell area $3.6 \mathrm{~m}^{2}$ within the neighbourhood and $30 \mathrm{~m}^{2}$ outside. The average cell width within the built area is $2.5 \mathrm{~m}$, as shown in Figure 9 . Three types of boundary conditions are used in the model (Figure 9). Boundary type 1 is a wall boundary condition which is prescribed along the levee and the canal bank, as well as all building walls. Boundary type 2 is a prescribed free surface elevation which is specified to be $8 \mathrm{~m}$ across both the upstream and downstream sections of the Deep Water Ship Canal. Boundary type 3 is a free outflow boundary condition. This condition is ensured by lowering artificially the mesh elevation by $4 \mathrm{~m}$ along the boundary and prescribing a Froude number in the normal direction $\mathbf{u} . \mathbf{n} / c=0.9$. An a posteriori check 
of the simulation results confirms that the flow conditions within the model remain hydraulically disconnected from those at the boundary at all times.

\section{Figure 9 about here}

Figure 9: Refined model. Model definition sketch and magnified view of the mesh. Boundary type 1: impervious. Boundary type 2: prescribed water level. Boundary type 3: free outflow.

Coarse grid models. A coarse grid was created to support application of the IP and DIP models. The grid consists of 2529 cells (Figure 10) with an average area of $345 \mathrm{~m}^{2}$ within the neighbourhood (to be compared with the average $3.6 \mathrm{~m}^{2}$ in the refined model). A combination of triangular and quadrilateral cell types were used to most efficiently span the domain and place vertices at the centroids of buildings, so model edges resolve the most narrow gaps between buildings to regulate conveyance $[30,22]$ and to ensure that $\phi_{\Gamma}<\phi_{\Omega}$. The mesh shown in Figure 10 is the result of manual processing. The boundary conditions in this model are identical to those in the refined model. As in the fine grid model, a zero bottom friction is assumed, therefore the bottom drag coefficient is set to zero and only the building drag coefficient is to be calibrated.

Model parametrization requires that the porosities $\phi_{\Omega}$ and $\phi_{\Gamma}$ be computed over each cell and each interface respectively. This is done by sampling the coarse model cells and interfaces. In each cell of the coarse mesh, a large number of points $(N=10,000)$ is generated with a uniform density so as to cover the entire cell. For each of these points, the refined model mesh is scanned to determine whether the point belongs to a building (thus lying outside the refined model domain) or is within the refined model mesh. The domain porosity $\phi_{\Omega}$ is computed as the ratio of the number $n$ of points lying within the refined model mesh to the total number $N, \phi_{\Omega}=n / N$. A similar procedure is used for the cell interfaces. The order of magnitude of the imprecision in the areal and connectivity porosities is therefore $10^{-4}$.

The porosity models were implemented using spatially uniform drag parameters and transient momentum dissipation parameters. The former were calibrated under steady state conditions, by comparison to fine-grid model predictions, and the latter were calibrated under transient conditions. Although the momentum dissipation model presented in this paper is not part of the original IP model, it was implemented here for the sake of model comparison. In this way, the IP and DIP models have the same number of calibration parameters. Benchmarking is thus free from model dimension-induced biases. Additional detail is provided in the following sections.

Figure 10 about here

Figure 10: Porosity model. View of the mesh.

\subsection{Model performance indicators}

Several model performance indicators are used. The first is the $L$-norm of the error between the porosity model and the fine grid simulation results. For a simulated variable $U$, the norm is defined as:

$$
L_{p}=\left[\frac{\sum_{i} \phi_{\Omega_{i}} A_{i}\left|U_{i}-\overline{U_{i}}\right|^{p}}{\sum_{i} \phi_{\Omega_{i}} A_{i}}\right]^{1 / p}, p>0
$$

where $A_{i}$ and $\phi_{\Omega i}$ are respectively the area and the areal porosity of the cell $i$ of the coarse model mesh, $U_{i}$ and $\overline{U_{i}}$ are respectively the values for $U$ over the cell $i$ computed by the porosity and the refined model. $\overline{U_{i}}$ is computed by averaging $U$ in all the cells of the refined mesh that belong to the cell $i$ of the porosity model. Three different norms are tested: the $L_{1}, L_{2}$ and $L_{\infty}$ norms, but only $L_{1}$ norms are reported hereafter because the $L_{2}$ trends were similar and the $L_{\infty}$ norm was found to be insensitive. 
The second indicator is the Flood Extent Agreement $F_{A}$, defined as

$$
\begin{gathered}
F_{A}=\frac{\sum_{i} \phi_{\Omega_{i}} A_{i}^{(I)}}{\sum_{i} \phi_{\Omega_{i}} A_{i}^{(U)}} \\
A_{i}^{(U)}=\left\{\begin{array}{ccc}
A_{i} & \text { if } & i \in A^{(U)} \\
0 & \text { if } & i \notin A^{(U)}
\end{array}, A_{i}^{(I)}=\left\{\begin{array}{ccc}
A_{i} & \text { if } & i \in A^{(I)} \\
0 & \text { if } & i \notin A^{(I)}
\end{array}\right.\right.
\end{gathered}
$$

where the superscripts $(I)$ and $(U)$ denote cells that make up the intersection and union, respectively, of the flooded area predicted by the refined model and porosity model. A flood extent agreement $F_{A}=1$ indicates that both models give identical flooded areas (thus the best possible agreement), while $F_{A}=0$ indicates that the flooded areas computed by both models have no intersection (thus the worst possible fit).

The third indicator, used for vector variables such as the flow velocity and unit discharge, is the polar histogram. The polar histogram for a vector field $\mathbf{w}$ is computed as follows. The 360 degrees angular range is divided into $N=36$ intervals. For each class $i=1, \ldots, N$ the following contribution is computed

$$
w_{i}=\sum_{j \in i} A_{j}\left\|\mathbf{w}_{j}\right\|, \quad W_{i}=\frac{w_{i}}{\sum_{i=1}^{N} w_{i}}
$$

where $j \in i$ is the set of cells in the model for which the direction of the flow velocity falls within the $i$ th direction interval, $A_{j}$ and $\mathbf{w}_{j}$ are respectively the area and the average value of the vector $\mathbf{w}$ in the computational cell $j$. The sum of the $W_{i}$ is therefore equal to unity and the polar histogram must be interpreted as a normalized contribution of the various flow directions.

\subsection{Steady-state analysis}

The classical shallow-water model and porosity model are first run for a period of $t=2400 \mathrm{~s}$ which establishes a steady state flow from the Deep Water Ship Channel across the urbanized development and over its outer perimeter (free overfall boundary condition). At a steady-state, the dissipation parameter $\mu=0$, so the role of drag coefficients on model accuracy can be isolated as in previous applications of porosity models $[31,30,22]$. The building drag coefficients in the $x$ - and $y$-directions for the IP and DIP models are assumed uniform over the neighbourhood and zero outside. Drag coefficient values between 0 and 0.025 were tested to identify the optimal parameter value (i.e., calibration).

Figure 11 shows the maps of the $L_{1}$ norm of the water depth error as a function of the building drag coefficients in the $x$ - and $y$-directions. Figure 11a and 11b show the $L_{1}$ norm of the water depth error for the IP and DIP models respectively. Errors for the IP model are slightly larger than for the DIP model, for example, IP errors range from $\sim 0.12-0.19 \mathrm{~m}$ while DIP errors range from $\sim 0.11-0.17$ $\mathrm{m}$. Figure 11 also shows that the sensitivity of each model to the drag coefficient is relatively small, at least within the range of parameter values considered here, compared to the sensitivity of the depth predictions to the porosity model (IP vs DIP). Calibrating the drag coefficient against the water depth leads to the optimal values shown in Table 5 . Table 5 also shows the corresponding $L_{1}$ error norms for the flow velocity and the unit discharges. The optimal value of the drag coefficient for IP and DIP yield water depth errors of 0.122 and $0.107 \mathrm{~m}$ respectively. From a practical perspective, depth errors of $0.10-0.15 \mathrm{~m}$ in a flood prediction would be considered acceptable given that aerial lidar data are typically characterized by errors of 0.10-0.015 m RMSE on hard surface such as concrete [29]. However, from a model development perspective, this error poses an opportunity for additional model improvements. The $L_{1}$-norms of the flow velocities and unit discharge are seen to be larger for the DIP model than for the IP model.

\section{Figure 11 about here}

Figure 11: Calibration of the building drag coefficient. L1-norm of the water depth error. Left: IP model. Right: DIP model. 


\section{Table 5 about here}

Table 5: Calibration of the building drag coefficient against the steady state solution. Optimal parameter sets.

Figure 12 shows the flow fields computed by the refined, IP and DIP models at $t=2400$ s. Figure 13 shows the polar histogram for the steady state flow velocity and unit discharge. It is visible from Figures 12 and 13 that the refined flow simulation exhibits two preferential directions along the main street directions. The polar histograms for the velocity and the unit discharge have very similar shapes. This indicates that the flow is aligned along the two preferential directions regardless of the water depth. Quite strikingly, averaging the refined simulation results onto the coarse grids used by the IP and DIP models does not eliminate this directional behaviour. This indicates that the flow field is strongly directional on the scale of the building block. Both the IP and DIP models fail to identify these preferential directions to a large extent. In the IP model, the histogram spreads rather evenly over the fourth quadrant (spanning the South to East directions), while in the DIP model the contribution of the $x$-velocity is exaggerated. The cells with a large $x$-velocity have small depths, therefore their contribution to the unit discharge histogram is comparatively small. This explains that the unit discharge histogram (Figure 13, lower right diagram) is more isotropic than the velocity histogram. Both the IP and DIP models overestimate the velocity and unit discharge in the diagonal direction.

\section{Figure 12 about here}

Figure 12: Calibration of the building drag coefficient. Unit discharge $(h \mathbf{u})$ and velocity vector fields $(\mathbf{u})$ for the steady state flow velocity and unit discharge at $t=2400 \mathrm{~s}$. Top: flow velocity. Bottom: unit discharge.

\section{Figure 13 about here}

Figure 13: Polar histograms for the steady state flow velocity and unit discharge at $t=2400$ s. Top: flow velocity. Bottom: unit discharge. "Refined simulation": fine grid simulation results averaged onto the coarse mesh used by the IP and DIP models.

\subsection{Transient analysis}

The preceding modelling scenario is now analysed at $t=120$ s when the levee-break wave front has penetrated the neighbourhood but has yet to reach the model boundary. Under transient conditions, the DIP model requires a dissipation parameter $\mu>0$, and here a calibration procedure is used to estimate its value using the fine grid solution as a benchmark. It is assumed that $\mu$ is uniform within the neighbourhood and zero outside. Given building layout, the principal directions of the tensor are the $x-$ and $y$-directions. Therefore, $\mu_{x y}=\mu_{y x}=0$ and only the coefficients $\mu_{x x}$ and $\mu_{y y}$ are calibrated assuming a range of 0 to 1 . The optimal parameter values that minimize the depth error ( $L_{1}$ norm) are given in Table6. The contrast between the IP and DIP models is striking. While the optimal $x$ - and $y$ - dissipation coefficients for the IP model are small (0.0 to 0.2), the optimal

$y$ - dissipation coefficient is 0.7 for the DIP model, while no $x$-momentum dissipation is required to reach optimal calibration. This is somewhat surprising in that the geometry of the streets is roughly the same in the $x$ - and $y$-directions. The structure of the neighbourhood thus clearly involves two principal directions. A possible explanation is that the wave propagation properties play a key role in reproducing the flow features and that the momentum dissipation tensor is of secondary importance. Another possible explanation is that the momentum dissipation model is incompletely parameterized (see the discussion in Section 5). This may also be seen as a confirmation of previous theoretical and experimental studies $[15,22]$ where anisotropy was identified as as a key feature of flow dynamics. 


\section{Table 6 about here}

Table 6: Calibration of the momentum dissipation coefficient against the transient solution at $t=120 \mathrm{~s}$. Optimal parameter sets.

Table 3 shows that the $L_{1}$ norm for the water depth and discharge per unit width is smaller in the DIP model than in the IP model. On the other hand, the $L_{1}$ norm of the flow velocity error is significantly larger in the DIP model than the IP model. This contrast in the performance with respect to $V$ and $h V$ is explained as follows: the DIP model makes a larger flow velocity error than the IP model in a limited number of cells. In these cells the water depth is very small, which contributes to a small error in terms of unit discharge. The flood extent agreement in the DIP model is also significantly better than for the IP model.

Figure 14 shows water depths at at $t=120$ s based on the refined model (Fig. 14a), the refined model coarsened to the scale of the porosity model (Fig. 14b), the IP model (Fig. 14c), and the DIP model with three combinations of dissipation parameters (Fig. 14d-e). This shows that an increase in $\mu_{y y}$ reduces spreading of flood water in the $y$ - direction and qualitatively improves the accuracy of the DIP prediction, compared with the coarsened fine grid prediction. Hence, inclusion of anisotropic dissipation parameters adds a second level of control over the spreading of flood water, in addition to the anisotropic porosity distribution resulting from the intersection of cell edges and building footprints. Note that the IP model with the parameter set $\left(\mu_{x x}, \mu_{y y}\right)=(0,0.7)$ gives $L_{1}(h)=0.082 \mathrm{~m}, L_{1}(V)=$ $0.69 \mathrm{~m} \mathrm{~s}^{-1}$ and $L_{1}(h V)=0.32 \mathrm{~m}^{2} \mathrm{~s}^{-1}$.

Figure 14 about here

Figure 14: Real world test case. Water depths simulated at $t=120$ s. $x-$ and $y-$ tick mark spacing $100 \mathrm{~m}$.

The unlikely parameter set $\left(\mu_{x x}=0, \mu_{y y}=0.7\right)$ for the DIP model is explained to some extent by the vector fields and the polar histograms in Figures 15-16. The flow velocity field simulated over the fine grid at $t=120 \mathrm{~s}$ exhibits the same two preferential directions as those observed at $t=2400 \mathrm{~s}$. The $y$-direction, that is associated with small depths, contributes much less to the histogram of the unit discharge. This strongly anisotropic wave propagation behaviour is preserved after averaging the fine grid simulation results onto the coarse grid. This again is an indication that the flow propagation pattern exhibits two preferential directions on the scale of the building block. The velocity and unit discharge fields computed by the IP model give too much weight to the $y$-direction, while the flow velocity computed by the DIP model give too much weight to the $x$-direction. The polar histogram for the unit discharge in the DIP model is very similar to that of the refined model. This may explain the smaller $L_{1}$ error in the water depths. Figure 15 confirms the $F_{A}$ values in Table 3 , in that the IP model slightly overestimates the extent of the flooded area compared to the DIP model.

\section{Figure 15 about here}

Figure 15: Unit discharge $(h \mathbf{u})$ and velocity vector fields $(\mathbf{u})$ simulated for $t=120 \mathrm{~s}$. Top: flow velocity. Bottom: unit discharge.

\section{Figure 16 about here}

Figure 16: Polar histograms of the flow velocity and unit discharge at $t=120 \mathrm{~s}$. Top: flow velocity. Bottom: unit discharge."Refined simulation": fine grid simulation results averaged onto the coarse mesh used by the IP and DIP models.

This may be attributed to several reasons: (i) the spatial scale of the perturbation (here the width of the dyke breach) is of the same order of magnitude as the size of the building block, which is 
practically relevant, but violates the scale separation principle (a basic requirement for upscaled, continuum-based model validity, [1,2]), (ii) the impinging wave propagates along one of the principal directions of the drag and momentum dissipation tensors, thus resulting in a poorly conditioned inverse problem, (iii) the momentum dissipation tensor model may be incomplete. In the present model, the tensor $\mu$ is assumed independent from the flow variables (water depth and flow velocities). It is easy to imagine, however, that the dissipation rate of the hydraulic transients described in Section 2 depends on the flow conditions. Besides, it may well be that the $2 \times 2$ tensor formulation (18) does not suffice to fully reproduce the flood dynamics. This point is detailed in the discussion section.

\section{Discussion}

There has been a debate as to whether the differential form of the porous shallow water equations is meaningful, even though it is clearly valuable for demonstrating wave propagation properties [30, 15]. Writing the equations in differential form and inferring a continuous solution should not be considered a problem if the modeller does not try to interpret the solution at a scale smaller than the typical dimension of the heterogeneities (typically, the building size). Groundwater flow modellers deal with a similar problem [36] in strongly heterogeneous aquifers where the REV does not exist, or, if it does, it is significantly larger than the computational cell size [3]. It is possible to refine shallow-water porosity model grids to minimize numerical truncation errors. However, mesh refinement undermines the primary goal of porosity models to radically reduce the computational cost of urban flood modelling, and calls for new methods of estimating porosity parameter distributions based on the intersection of mesh cells and edges with building footprints. The authors acknowledge that the implications of mesh design in terms of numerical accuracy have not been fully explored for the IP and DIP model and that further research work is needed along this line.

The unusual transient momentum dissipation model presented in Section 2.3 is clearly superior to energy dissipation models usually found in the literature. In particular, it allows the large scale selfsimilar character of solutions to be preserved, while energy dissipation models do not. Nevertheless, the computational examples shown in Sections 3.1 and 3.3 indicate that further effort is needed to make the parameterization of this model fully explicit. Obviously, the dissipation tensor depends not only on the geometry, but also on the hydraulic field. Indeed, while the computational examples in Sections 3.1 and 3.3 use the same geometry, the optimum value is $\mu_{x x}=0.156$ in Section 3.1 and $\mu_{x x}=0.48$ in Section 3.3. Likewise, a strongly anisotropic parameter set $\left(\mu_{x x}=0, \mu_{y y}=0.7\right)$ is obtained in the field scale test case (Section 4) over a model with almost identical $x$ - and $y$-geometries. More research will be needed to characterize the relationship between the dissipation tensor and the hydraulic variables. Another path for further research should consist in exploring a wider range of urban geometries (in terms of building alignment, spacing, street orientation, etc.) than those explored in the present paper.

The DIP and IP models include parameters that cannot be measured and therefore must be inferred. Whereas both models require a drag parametrization for buildings, the DIP also introduces momentum dissipation parameters that allow for additional control over flood spreading. If we assume that in any application of porosity models, a fine grid solution is needed to support calibration of drag coefficients, then the key difference between the DIP and original IP models is the number of parameters that need to be calibrated, and thus the number of porosity model simulations (trials or functional calls) needed to find optimal parameter values. One may question the value of a porosity model flood simulation if a fine grid model is nevertheless required; benefits arise when large numbers of scenarios are run to characterize model uncertainties (i.e, Monte Carlo methods) and when test cases on a relatively small domain can be used to to develop functional forms for porosity model parameters that can be extrapolated over large domains. Additional research is needed to pursue these possibilities.

When both the building drag and momentum dissipation models are incorporated in both the IP and DIP models, the DIP model gives more accurate results than the IP model. It is also noted that the DIP model is more accurate than the IP model in idealized 1D tests when the transient momentum dissipation is not activated, i.e., $\mu=0$. This indicates that the discharge closure model proposed in 
Subsection 2.2 is essential to an accuracte representation of wave propagation properties.

The two-dimensional application presented in Section 4.4 shows that neither the IP nor the DIP models achieve a satisfactory description of the anisotropic structure of the velocity distribution in the transient phase. The field scale test presented in Section 4 shows that the directional behaviour of the street network acts on a scale at least as large as that of the block scale. Averaging the flow field on the scale of a building block might have been expected to yield a rather isotropic velocity vector field. This is not the case, as the polar histograms in Figure 16 indicate. At any rate, the unlikely optimal parameter sets obtained from the calibration of both models (zero $y$-drag coefficient for the IP model, zero $x$-momentum dissipation for the DIP model) seem to point towards an inadequate model structure. This preliminary conclusion is supported by previously reported work on the consequences of attempting to calibrate overland flow models with inappropriate structures [19]. Bearing in mind that the IP model was specifically designed to address anisotropy issues [30], the reason for this limitation of the IP and DIP models is not clear at this stage.

Field studies with measurements of flood extent, flood depth and flood velocities are needed to deepen understanding of the performance of urban flood models in general, and porosity models in particular. Sensitivity analyses of the one- and two-dimensional shallow water equations $[16,17]$ show that one- and two-dimensional shallow water models behave very differently with respect to model geometry, parameters and boundary conditions. It should thus not come as a surprise that the DIP model exhibits excellent performance in one-dimensional test cases while showing moderate success in two-dimensional flow simulations. It has been shown at the laboratory scale that a combination of physical measurements, fine grid model predictions, and porosity model predictions can be used to distinguish between structural model errors in the shallow-water equations, scaling errors associated with use of relatively coarse grid, and porosity model errors [22]. However, field-scale performance is the true test of these models and in this context there are many additional sources of uncertainty beyond what is encountered at the laboratory scale such as uncertainty in topography, flow volumes, flood control infrastructure such as storm drains and pumps, and the physical behaviour of buildings such as their capacity to store and convey flood water, e.g., through doors and windows. Previous work on shallow-water models suggests that topographic errors and flood volumes are the primary controls on urban flood model accuracy [5, 12], so a key remaining issue in the development of porous shallow-water models is whether and to what extent porosity models errors presently serve to limit model accuracy.

\section{Conclusions}

The Dual Integral Porosity (DIP) model presented herein achieves closure of the previously introduced Integral Porosity (IP) model by defining an edge-based model solution (boundary of computational cell) in addition to a cell-based model solution (interior of a computational cell). The DIP model results in a modification of the mass and momentum fluxes compared to the IP model, as well as the wave propagation speeds. A wave propagation speed analysis of the DIP model shows that problem well-posedness requires that the storage porosity be greater than or equal to the conveyance porosity. Several verification test cases including a positive wave, a negative wave and a dam-break wave clearly demonstrate a major accuracy advantage of the DIP model over the IP model when predicting the flow field, including flow depth, flow velocity and the position of shock waves even without the proposed transient dissipation model. Furthermore, the transient momentum dissipation scheme proposed for the DIP model is shown to exert a significant influence on the propagation properties of positive waves. This model contributes to increase the response range of the DIP model compared to the original IP model. Furthermore, the transient dissipation model introduces another level of control for improving model accuracy at the expense of an additional parameter that must be calibrated.

The DIP model includes a new, transient momentum dissipation term that acts directly on fluxes, in contrast to conventional momentum dissipation terms (i.e., bottom friction) that appear as a sink term. Verification test cases support the use of dissipation only for positive waves defined by $\partial_{t} h>0$. 
The transient dissipation parameter(s) can be identified through a calibration procedure using a fine scale shallow-water model as a reference. In two dimensions, this is introduced as a $2 \times 2$ tensor.

Field scale testing shows that the DIP model can achieve smaller errors in flood depth and discharge per unit with, and better flood extent accuracy, compared to the IP model. Additionally, field-scale testing shows that transient momentum dissipation introduces an important control for shaping the directionality of flood spreading within arrays of buildings that obstruct flow. Hence, the DIP offers greater versatility for field-scale modelling than the IP model in the sense that there is greater control over model solutions. Nevertheless, both the DIP and IP models fail to some extent to reproduce the preferential directions of the flow field in the transient phase. The practice of shallow-water porosity model calibration and application is at its infancy, and thus more work is needed to provide guidance for accurate and efficient modelling and to deepen understanding of the trade-offs between model structure (including the number of calibration parameters), mesh design and model performance at field scale.

\section{Acknowledgements}

The authors with to thank the City of West Sacramento, California, USA for graciously providing aerial lidar data for use in this study. Participation by BFS and JES on this research project was made possible by a grant from the National Science Foundation (DMS-1331611), who support is gratefully acknowledged.

\section{References}

[1] Auriault JL. Effective macroscopic description for heat conduction in periodic composites. Internation Journal of Heat and Mass Transfe 1983;26(6):861-869.

[2] Auriault JL. Heterogeneous medium. Is an equivalent macroscopic description possible? International Journal of Engineering Science 1991;29(7):785-795.

[3] Baghbanan A, Jing L. Hydraulic properties of fractured rock masses with correlated length and aperture. Int J Rock Mech Mining Sci 2007;44:704-19.

[4] Bates PD. Development and testing of a sub-grid scale model for moving boundary hydrodynamic problems in shallow water. Hydrol Process 2000;14:2073-2088.

[5] Bates PD. Integrating remote sensing data with flood inundation models: how far have we got? Hydrological Processes 2012;26(16):2515-2521.

[6] Bear J. Dynamics of fluids in porous media. New York: Dover Publications Inc.;1998.

[7] Benkhaldoun F, Elmahi I, Moumna A, Seaid M. A non-homogeneous Riemann solver for shallow water equations in porous media. Applicable Analysis, accepted, DOI: 10.1080/00036811.2015.1067304.

[8] Cea L, Vazquez-Cendon ME. Unstructured finite volume discretization of two-dimensional depthaveraged shallow water equations with porosity. Int J Numer Methods Fluids 2010;63:903-30.

[9] Defina A. Two-dimensional shallow flow equations for partially dry areas. Water Resour Res 2000;36:3251-64.

[10] Defina A, D'Alpaos L, Mattichio B. A new set of equations for very shallow water and partially dry areas suitable to 2D numerical models. In: Molinaro P, Natale L, editors. Proceedings of the specialty conference "Modelling flood propagation over initially dry areas". NY: Am. Soc. Civ. Eng.; 1994. p. 82-101. 
[11] Finaud-Guyot P, Delenne C, Lhomme J, Guinot V, Llovel C. An approximate-state Riemann solver for the two-dimensional shallow water equations with porosity. International Journal for Numerical Methods in Fluids 2010;62:1299-1331.

[12] Gallegos HA, Schubert JE, and Sanders BF. Two-dimensional, high-resolution modeling of urban dam-break flooding: a case study of Baldwin Hills, California, Advances in Water Resources 2009;32:1323-1335.

[13] Gallien TW, Schubert JE and Sanders BF. Predicting tidal flooding of urbanized embayments: A modeling framework and data requirements. Coastal Engineering 2011;58(6):567-577.

[14] Guinot V. Wave Propagation in Fluids. Models and numerical techniques. 2nd Edition. ISTE, 2010.

[15] Guinot V. Multiple porosity shallow water models for macroscopic modelling of urban floods. Advances in Water Resources 2012;37:40-72.

[16] V. Guinot, Cappelaere B. Sensitivity equations for the 1D shallow water equations. Practical application to model calibration. Journal of Hydrologic Engineering 2009;14(8):858-861.

[17] Guinot V, Cappelaere B. Sensitivity analysis of 2D steady state shallow water flow. Application to free surface flow model calibration. Advances in Water Resources 2009;32:540-560.

[18] Guinot V, Delenne C. Macroscopic modelling of urban floods. La Houille Blanche 2014;6;19-25.

[19] Guinot V, Gourbesville P. Calibration of physically-based models: back to basics? Journal of Hydroinformatics 203;05.4:233-244.

[20] Guinot V, Soares-Frazão S. Flux and source term discretisation in two-dimensional shallow water models with porosity on unstructured grids. International Journal for Numerical Methods in Fluids 2006;50:309-345.

[21] Hervouët JM, Samie R, Moreau B. Modelling urban areas in dam-break floodwave numerical simulations. In: Proceedings of the international seminar and workshop on rescue actions based on dambreak flow analysis, Seinâjoki, Finland, 1-6 October 2000.

[22] Kim B, Sanders BF, Famiglietti JS, Guinot V. Urban flood modeling with porous shallow-water equations: A case study of model errors in the presence of anisotropic porosity. Journal of Hydrology 2015;523:680-692.

[23] Lax PD. Hyperbolic systems of conservation laws II. Communications on Pure and Applied Mathematics 1957;10:537-566.

[24] Lhomme J. One-dimensional, two-dimensional and macroscopic approaches to urban flood modelling. PhD thesis (in French), Université Montpellier 2, 2006.

[25] Ludy J, Kondolf GM. Flood risk perception in lands "protected" by 100-year levees. Natural Hazards 2012;61:829-842.

[26] Nepf HM. Drag, turbulence, and diffusion in flow through emergent vegetation. Wtare Resources Research 1999;35:479-489.

[27] Özgen I, Liang D, Hinkelmann R. Shallow water equations with depth-dependent anisotropic porosity for subgrid-scale topography. Applied Mathematical Modeling 2015, http://dx.doi.org/10.1016/.j.apm.2015.12.012

[28] Özgen I, Liang D, Hinkelmann R. Urban flood modeling using shallow water equations with depth-dependent anisotropic porosity. Journal of Hydrology 2016;54:1165-1184. 
[29] Sanders BF. Evaluation of on-line DEMs for flood inundation modeling. Advances in Water Resources 2007;30 (8):1831-1843.

[30] Sanders BF, Schubert JE, Gallegos HA. Integral formulation of shallow water equations with anisotropic porosity for urban flood modelling. J Hydrol 2008;362:19-38.

[31] Schubert JE, Sanders BF. Building treatments for urban flood inundation models and implications for predictive skill and modeling efficiency. Advances in Water Resources 2012;41:49-64.

[32] Guinot V, Soares-Frazão S. An eigenvector-based linear reconstruction scheme for the shallow water equations on two-dimensional unstructured meshes. International Journal for Numerical Methods in Fluids 2007;53:23-55.

[33] Soares-Frazao S, Lhomme J, Guinot V, Zech Y. Two-dimensional shallow water models with porosity for urban flood modelling. Journal of Hydraulic Research 2008;46(1):45-64.

[34] Stone B M, Shen H T. Hydraulic resistance of flow in channels with cylindrical roughness. Journal of Hydraulic Engineering, 2002, 128(5): 500-506

[35] Testa G, Zuccala D, Alcrudo F, Mulet J, Soares-Frazao S. Flash flood flow experiment ni a simplified urban district. Journal of Hydraulic Research 2007;45 (Extra Issue):37-44.

[36] Wen XH, Gomez-Hernandez JJ. Upscaling hydraulic conductivities in heterogeneous media: an overview. J Hydrol 1996;183:ix-xxxii. 


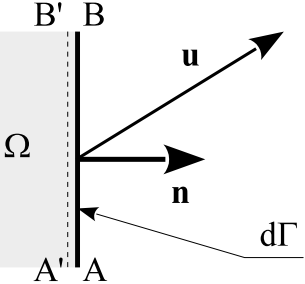




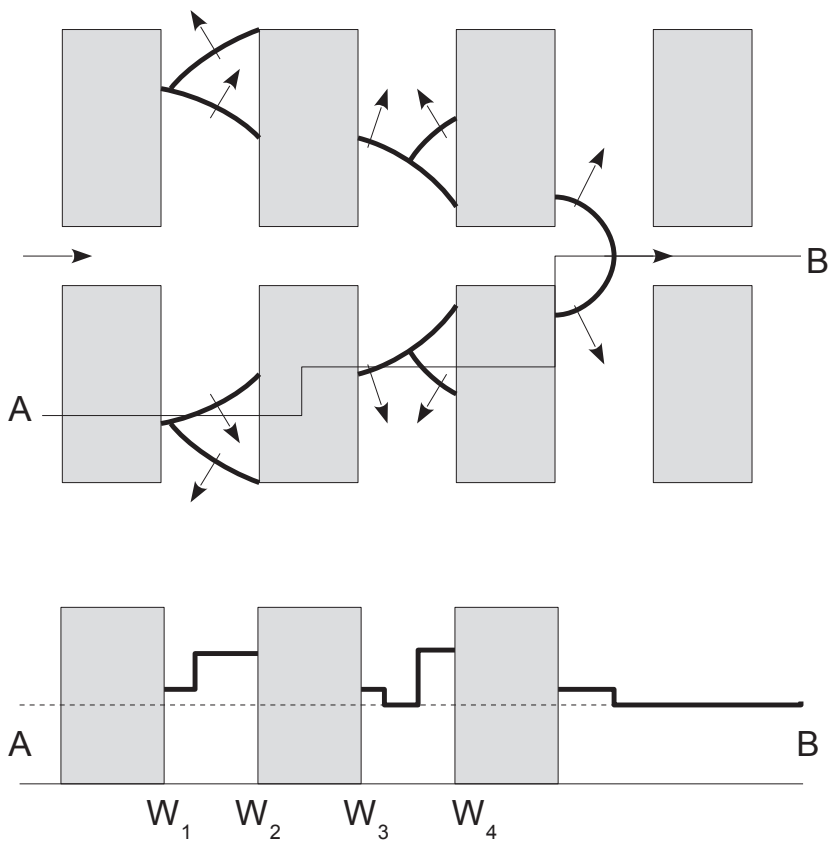




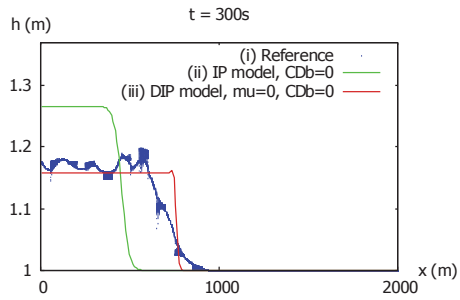

$$
h(m) \quad t=300 s
$$
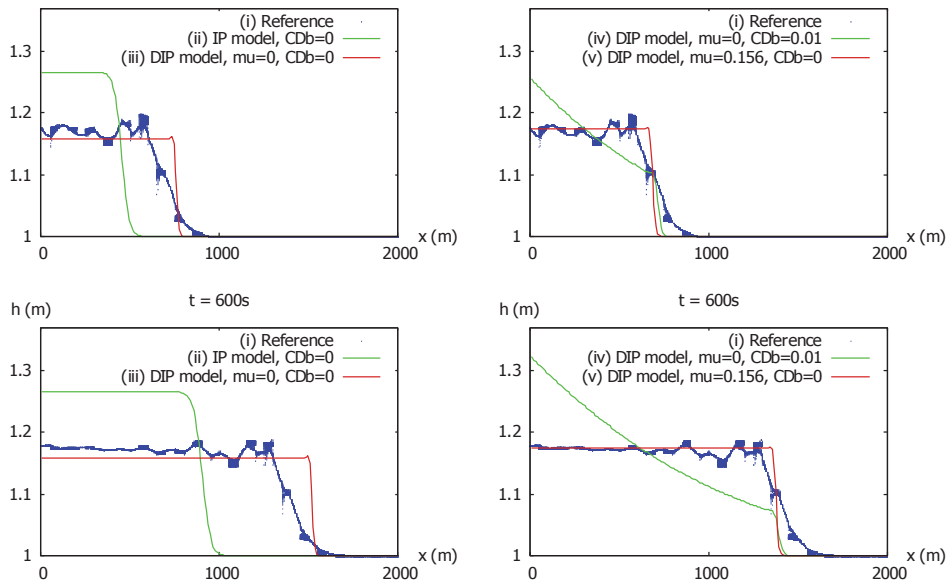

$$
h(m) \quad t=600 s
$$

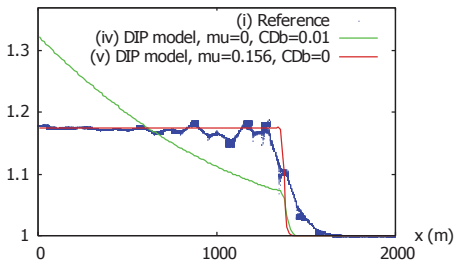




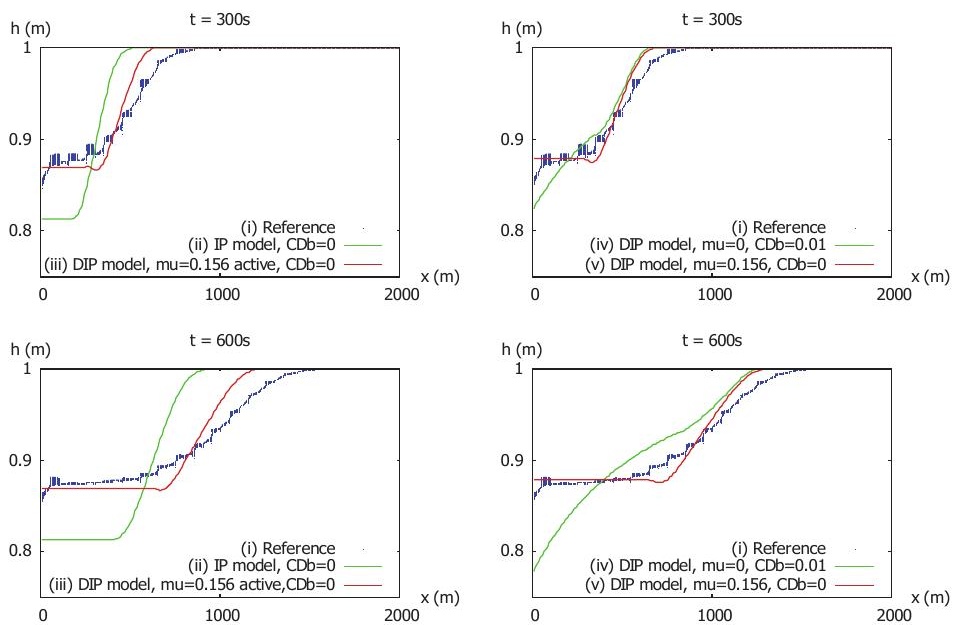



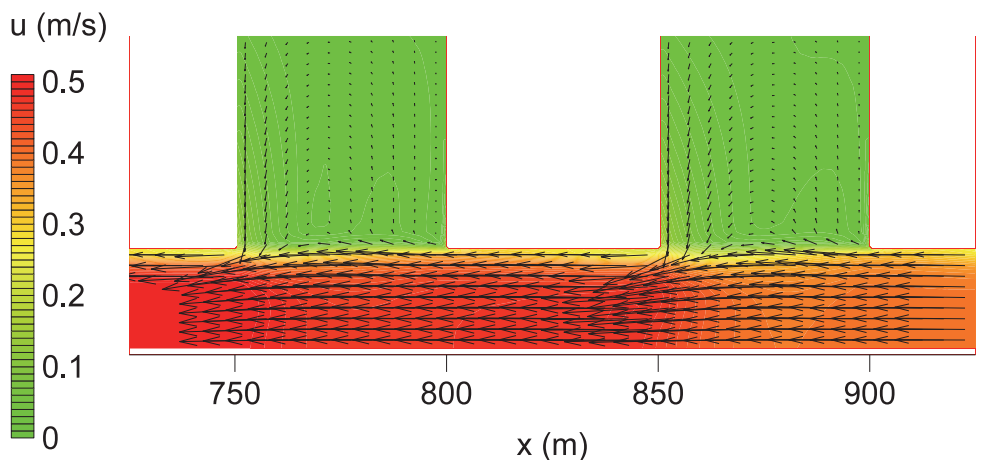

$x(m)$ 

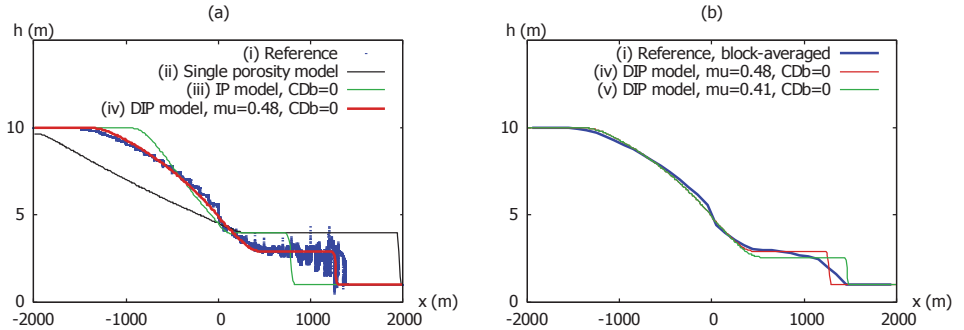

(c)
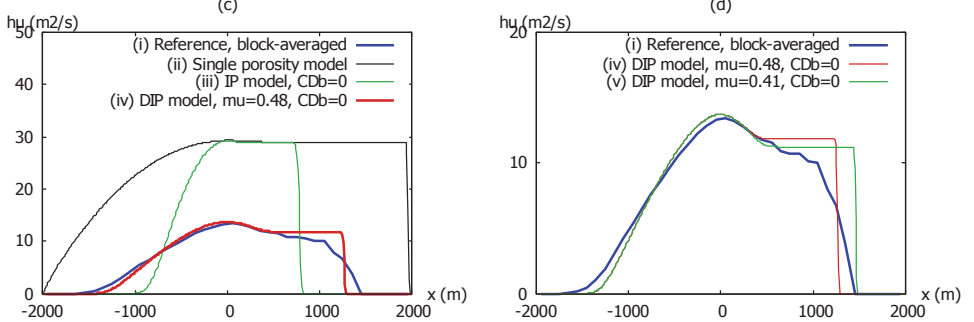


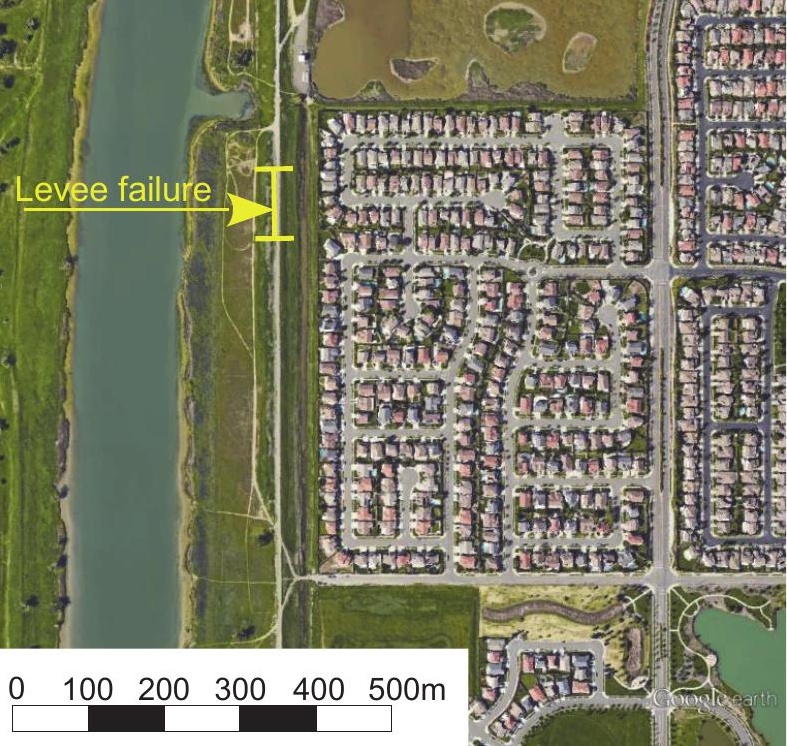




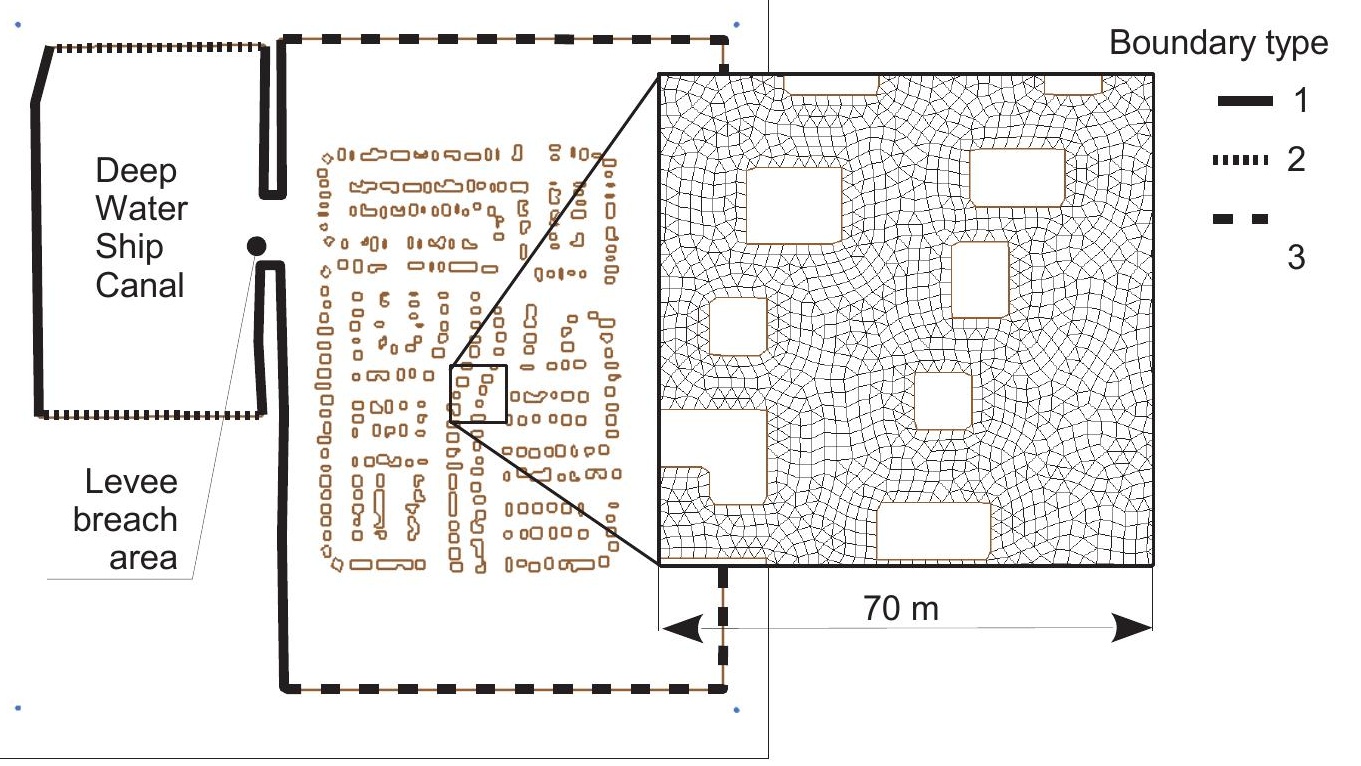




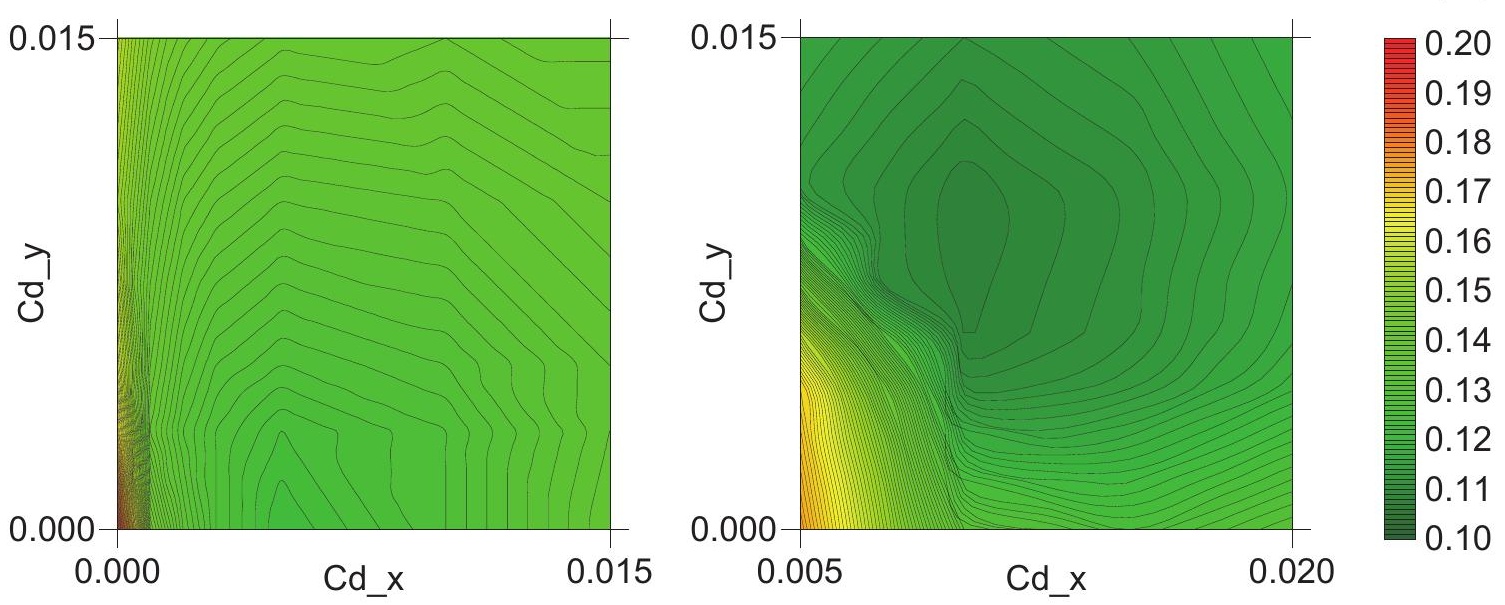


Refined simulation velocity

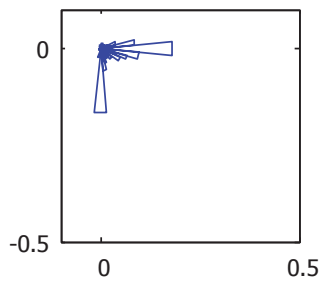

Refined simulation unit discharge

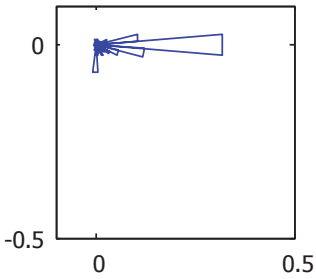

IP model velocity

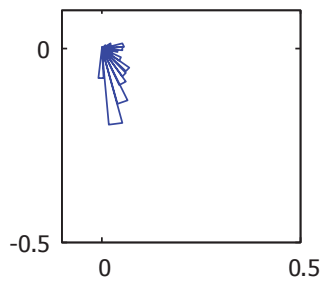

IP model unit discharge

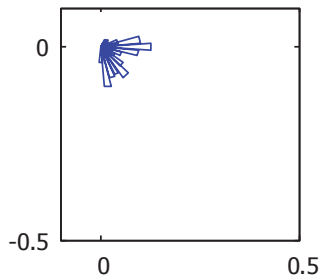

DIP model velocity

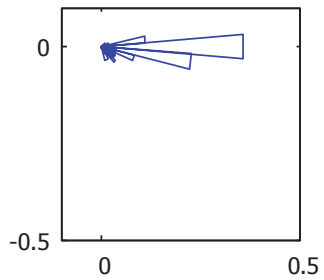

DIP model unit discharge

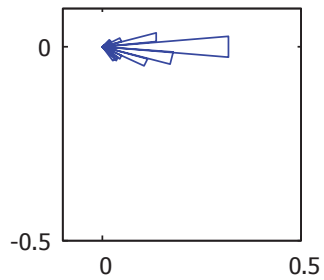




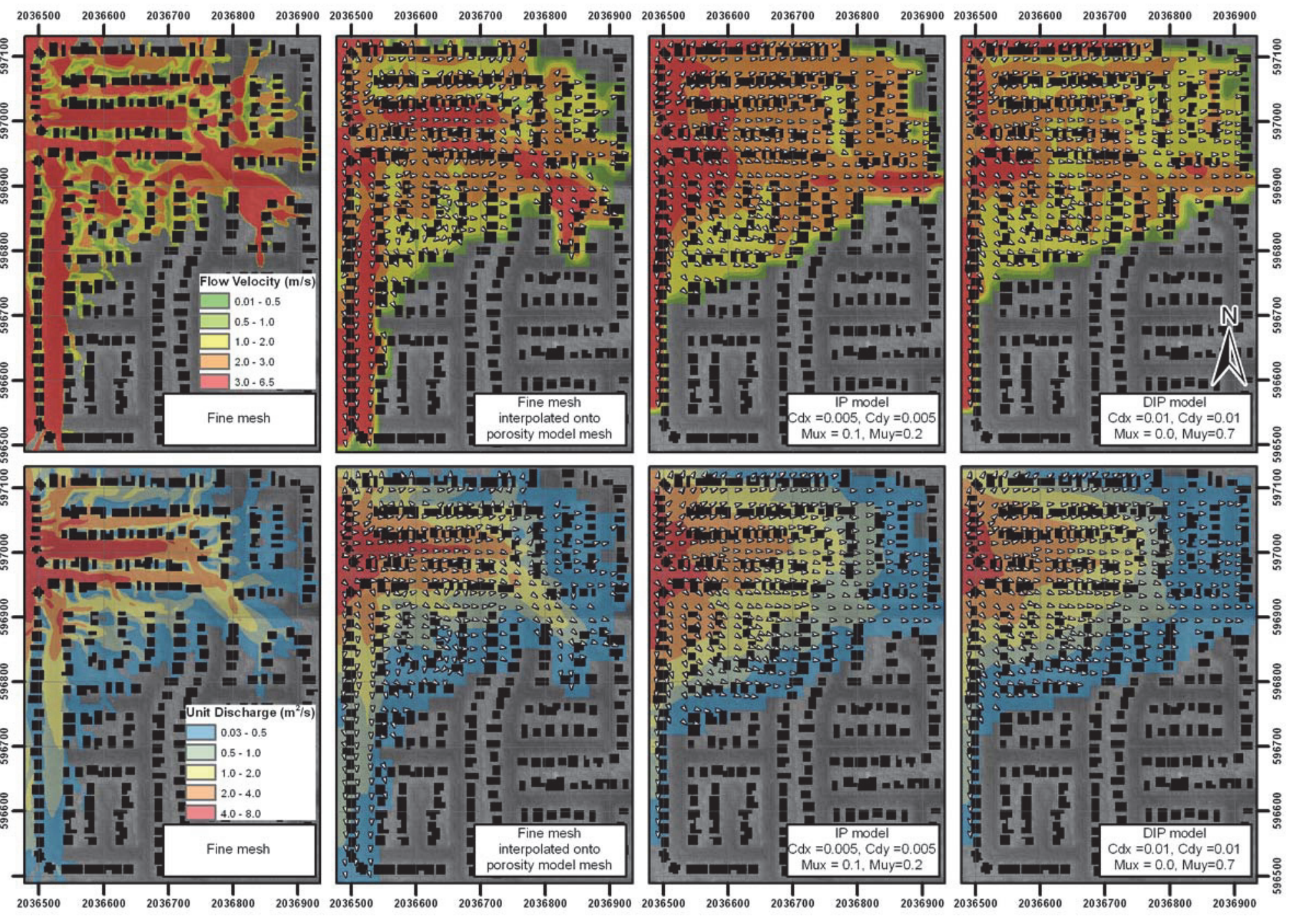




\section{Tables}

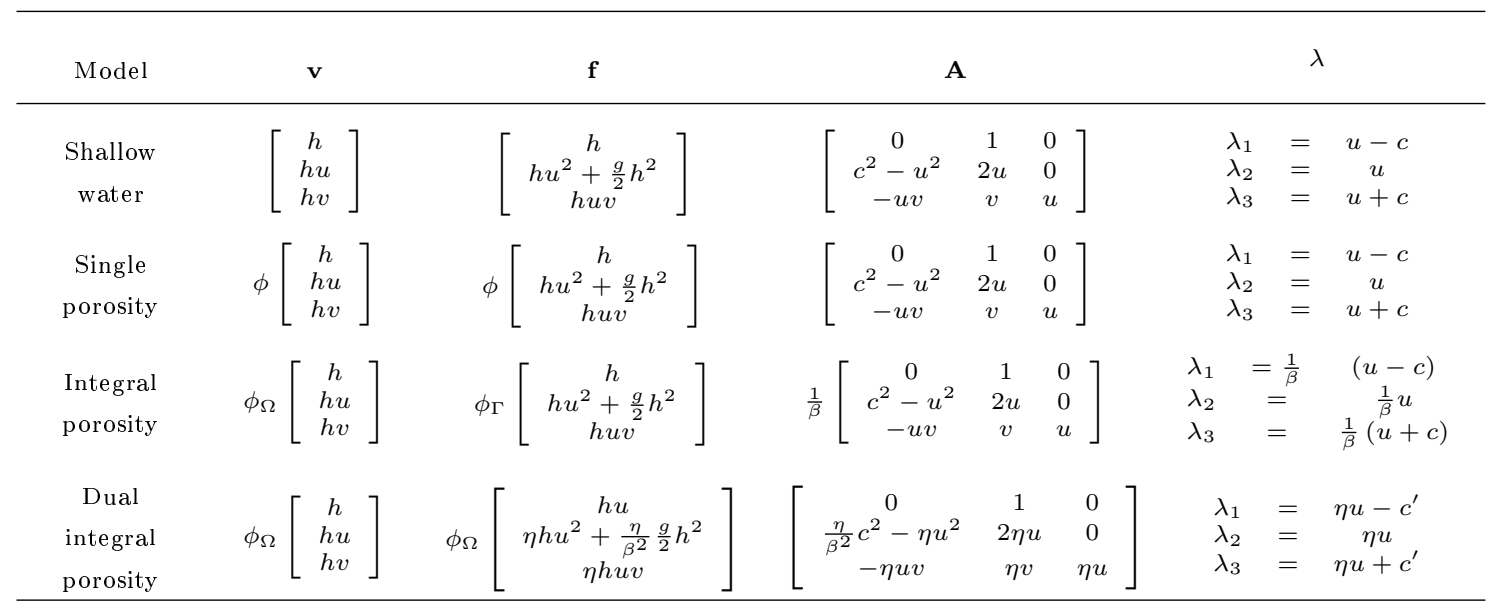

Table 1: Summary of the various formulations. For the sake of conciseness, only the onedimensional version is presented. Note: $c \equiv(g h)^{1 / 2}, c^{\prime} \equiv \eta^{\frac{1}{2}}\left[(\eta-1) u^{2}+\frac{c^{2}}{\beta^{2}}\right]^{\frac{1}{2}}$.

\begin{tabular}{ccccccc}
\hline Model & $L_{1}(h)(\mathrm{m})$ & $L_{1}(V)(\mathrm{m} / \mathrm{s})$ & $L_{1}(h V)\left(\mathrm{m}^{2} / \mathrm{s}\right)$ & $F_{A}$ & Optimal $C_{D, x}^{b}$ & Optimal $C_{D, y}^{b}$ \\
\hline IP & 0.122 & 0.63 & 0.39 & $100 \%$ & $5 \times 10^{-3}$ & 0 \\
DIP & 0.107 & 0.71 & 0.43 & $100 \%$ & $10^{-2}$ & $10^{-2}$ \\
\hline
\end{tabular}

Table 2: Calibration of the building drag coefficient against the steady state solution. Optimal parameter sets.

\begin{tabular}{ccccccc}
\hline Model & $L_{1}(h)(\mathrm{m})$ & $L_{1}(V)(\mathrm{m} / \mathrm{s})$ & $L_{1}(h V)\left(\mathrm{m}^{2} / \mathrm{s}\right)$ & $F_{A}$ & Optimal $\mu_{x x}$ & Optimal $\mu_{y y}$ \\
\hline IP & 0.081 & 0.12 & 0.32 & $77 \%$ & 0.1 & 0.2 \\
DIP & 0.067 & 0.84 & 0.26 & $91 \%$ & 0.0 & 0.7 \\
\hline
\end{tabular}

Table 3: Calibration of the momentum dissipation coefficient against the transient solution at $t=120$ s. Optimal parameter sets. 


\section{Tables}

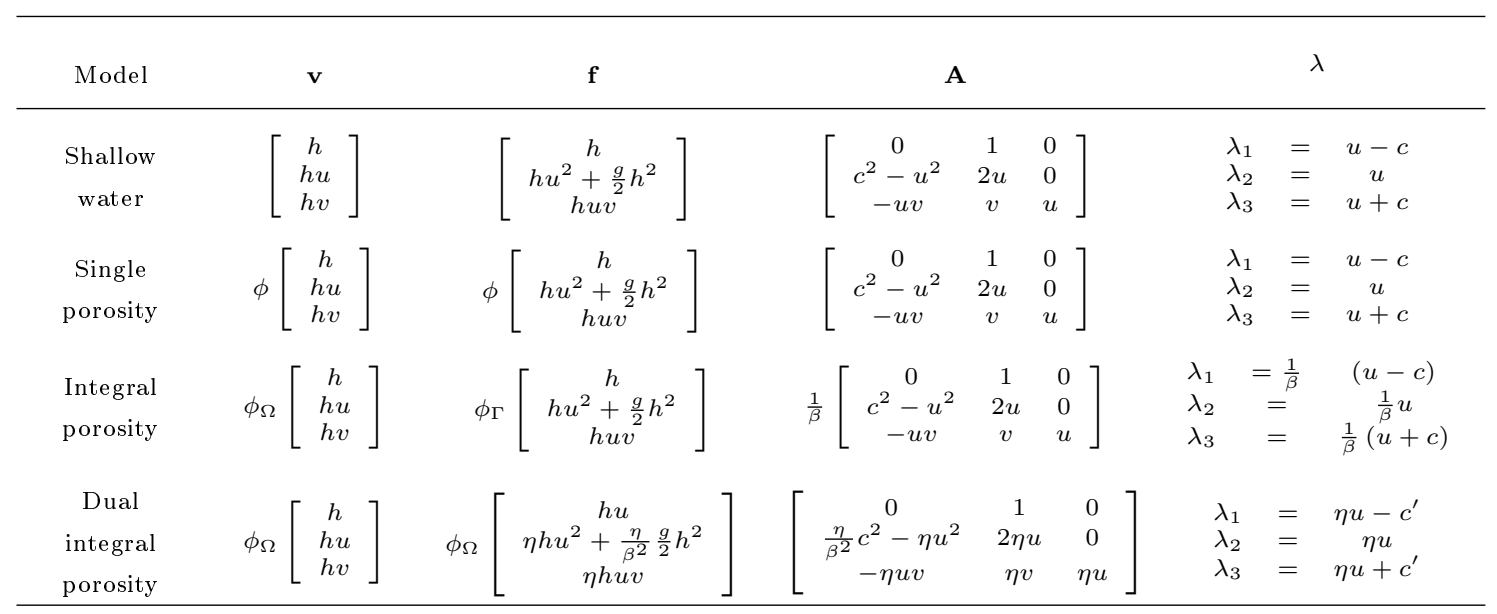

Table 1: Summary of the various formulations. For the sake of conciseness, only the onedimensional version is presented. Note: $c \equiv(g h)^{1 / 2}, c^{\prime} \equiv \eta^{\frac{1}{2}}\left[(\eta-1) u^{2}+\frac{c^{2}}{\beta^{2}}\right]^{\frac{1}{2}}$.

\begin{tabular}{cccccc}
\hline Simulation & (i) & (ii) & (iii) & (iv) & (v) \\
\hline Model & SW & IP & DIP & DIP & DIP \\
$C_{D}^{b}$ & NA & $0(\mathrm{~F})$ & $0(\mathrm{~F})$ & $0.01(\mathrm{C})$ & $0(\mathrm{~F})$ \\
$\mu_{x x}$ & NA & NA & $0(\mathrm{~F})$ & $0(\mathrm{~F})$ & $0.156(\mathrm{C})$ \\
\hline
\end{tabular}

Table 2: Propagation of a positive wave. Overview of simulations. C: parameter is calibrated; F: parameter is fixed; (NA): Not Applicable because the parameter does not exist in the model.

\begin{tabular}{cccccc}
\hline Simulation & (i) & (ii) & (iii) & (iv) & (v) \\
\hline Model & SW & IP & DIP & DIP & DIP \\
$C_{D}^{b}$ & (NA & $0(\mathrm{~F})$ & $0(\mathrm{~F})$ & $0.01(\mathrm{C})$ & $0(\mathrm{~F})$ \\
$\mu_{x x}$ & NA & NA & $0.156(\mathrm{C})$ & 0 & NA \\
\hline
\end{tabular}

Table 3: Propagation of a negative wave. Overview of simulations. C: parameter is calibrated; F: parameter is fixed; NA: Not Applicable because the parameter does not exist in the model. 


\begin{tabular}{cccccc}
\hline Simulation & (i) & (ii) & (iii) & (iv) & (v) \\
\hline Model & SW & SP & IP & DIP & DIP \\
$C_{D}^{b}$ & NA & NA & $0(\mathrm{~F})$ & $0(\mathrm{~F})$ & $0(\mathrm{~F})$ \\
$\mu_{x x}$ & NA & NA & NA & 0.48 & 0.41 \\
\hline
\end{tabular}

Table 4: Urban dam-break problem. Overview of simulations. C: parameter is calibrated; F: parameter is fixed; NA: Not Applicable because the parameter does not exist in the model.

\begin{tabular}{ccccccc}
\hline Model & $L_{1}(h)(\mathrm{m})$ & $L_{1}(V)(\mathrm{m} / \mathrm{s})$ & $L_{1}(h V)\left(\mathrm{m}^{2} / \mathrm{s}\right)$ & $F_{A}$ & Optimal $C_{D, x}^{b}$ & Optimal $C_{D, y}^{b}$ \\
\hline IP & 0.122 & 0.63 & 0.39 & $100 \%$ & $5 \times 10^{-3}$ & 0 \\
DIP & 0.107 & 0.71 & 0.43 & $100 \%$ & $10^{-2}$ & $10^{-2}$ \\
\hline
\end{tabular}

Table 5: Calibration of the building drag coefficient against the steady state solution. Optimal parameter sets.

\begin{tabular}{ccccccc}
\hline Model & $L_{1}(h)(\mathrm{m})$ & $L_{1}(V)(\mathrm{m} / \mathrm{s})$ & $L_{1}(h V)\left(\mathrm{m}^{2} / \mathrm{s}\right)$ & $F_{A}$ & Optimal $\mu_{x x}$ & Optimal $\mu_{y y}$ \\
\hline IP & 0.081 & 0.12 & 0.32 & $77 \%$ & 0.1 & 0.2 \\
DIP & 0.067 & 0.84 & 0.26 & $91 \%$ & 0.0 & 0.7 \\
\hline
\end{tabular}

Table 6: Calibration of the momentum dissipation coefficient against the transient solution at $t=120 \mathrm{~s}$. Optimal parameter sets. 